\title{
OPEN High yield synthesis of graphene quantum dots from biomass waste as a highly selective probe for $\mathrm{Fe}^{3+}$ sensing
}

\begin{abstract}
Aumber Abbas ${ }^{1}$, Tanveer A. Tabish², Steve J. Bull ${ }^{1}$, Tuti Mariana Lim ${ }^{3 凶}$ \& Anh N. Phan ${ }^{1 凶}$
Graphene quantum dots (GQDs), a novel type of zero-dimensional fluorescent materials, have gained considerable attention owing to their unique optical properties, size and quantum confinement. However, their high cost and low yield remain open challenges for practical applications. In this work, a low cost, green and renewable biomass resource is utilised for the high yield synthesis of GQDs via microwave treatment. The synthesis approach involves oxidative cutting of short range ordered carbon derived from pyrolysis of biomass waste. The GODs are successfully synthesised with a high yield of over $84 \%$, the highest value reported to date for biomass derived GQDs. As prepared GQDs are highly hydrophilic and exhibit unique excitation independent photoluminescence emission, attributed to their single-emission fluorescence centre. As prepared GQDs are further modified by simple hydrothermal treatment and exhibit pronounced optical properties with a high quantum yield of 0.23. These modified GQDs are used for the highly selective and sensitive sensing of ferric ions $\left(\mathrm{Fe}^{3+}\right)$. A sensitive sensor is prepared for the selective detection of $\mathrm{Fe}^{3+}$ ions with a detection limit of as low as $2.5 \times 10^{-6} \mathrm{M}$. The utilisation of renewable resource along with facile microwave treatment paves the way to sustainable, high yield and cost-effective synthesis of GQDs for practical applications.
\end{abstract}

Graphene quantum dots (GQDs), as a zero-dimensional derivates of graphene, have ignited tremendous research interests in recent years ${ }^{1-6}$. Due to the prominent quantum confinement ${ }^{6}$ and edge effects ${ }^{7}$, GQDs display excellent optical and electrical properties. GQDs offer significant advantages of low cost, high water solubility, stable fluorescence, tunable bandgap, low toxicity and good biocompatibility ${ }^{8-11}$, and thus making them a legitimate competitor to the traditional semiconductor quantum dots (QDs) (e.g. ZnS, TiO, CdSe, CdS, CdTe), which are expensive, cytotoxic, and show low-biocompatibility ${ }^{12}$. Owing to their unique properties, GQDs exhibit substantial potential for a wide range of promising applications in catalysis, sensors, bio-imaging, medical diagnosis, optoelectronics, and energy storage devices ${ }^{10,11,13-15}$. Therefore, an easy, cost-effective and high yield synthesis of GQDs remains an unmet need for their real-world application.

To date, several carbon-based resources have been used to synthesise GQDs, such as graphite flakes ${ }^{16}$, carbon nanotubes ${ }^{17}$, graphene ${ }^{18}$, carbon fibre ${ }^{19}$, coal $^{20}$ and others ${ }^{21,22}$. A number of synthesis techniques have been adopted such as hydrothermal ${ }^{18,23}$, electrochemical ${ }^{24,25}$, sonochemical ${ }^{26}$, solvothermal ${ }^{11,27,28}$, laser ablation ${ }^{29}$, microwave cutting ${ }^{30}$, etc. Among these approaches, microwave treatment is indeed beneficial due to its uniform and rapid heating, thereby leading to extremely short reaction time ${ }^{31}$. The utilisation of microwave treatment has been reported for the rapid synthesis of GQDs ${ }^{30,32}$. However, most of these reports either used expensive precursor materials, complex processes, highly toxic chemicals or gave very low product yield. For example, Li et al. synthesised greenish-yellow GQDs from graphene oxide to generate a product yield of $8 \%{ }^{30}$. Wang et al. reported the synthesis of white light emitting GQDs by two step microwave assisted hydrothermal approach that involved large amount of corrosive acids and required $14 \mathrm{~h}$ reaction time ${ }^{32}$. Shin et al. reported the synthesis of GQDs with relatively good yield, but the process involved large amount of corrosive and toxic chemicals $\left(\mathrm{H}_{2} \mathrm{SO}_{4}\right.$, $\left.\mathrm{KMnO}_{4}\right)^{33}$. In another report, Nair et al. synthesised GQDs by the microwave treatment of graphene oxide using strong oxidizing agent $\left(\mathrm{KMnO}_{4}\right)^{34}$. Thus, an easy, rapid, convenient, cost effective, and environmentally friendly approach for the high yield synthesis of GQDs from low cost precursors is highly demanded.

\footnotetext{
${ }^{1}$ School of Engineering, Newcastle University, Newcastle upon Tyne NE1 7RU, UK. ${ }^{2}$ UCL Cancer Institute, University College London, London WC1E 6DD, UK. ${ }^{3}$ School of Civil and Environmental Engineering, Nanyang Technological University, Singapore 639798, Singapore. ${ }^{\circledR}$ email:TMLim@ntu.edu.sg; anh.phan@ncl.ac.uk
} 
Biomass waste, which is green, cheap, abundant, easily available and rich in carbon, can be considered as a potential precursor for the synthesis of GQDs. Moreover, the disposal and recycling of biomass waste is becoming a major challenge of the modern world. Thus, the use of biomass waste would solve the recycling problem as well as fulfil the need for a cost-effective precursor for the synthesis of GQDs. Herein, biomass waste is proposed as a cheap and sustainable precursor for the significant high-yield production of GQDs for the first time, through a simple microwave approach. Interestingly, the hydrothermally modified form of these GQDs exhibit pronounced optical properties and are utilised in a selective and sensitive detection of ferric ions $\left(\mathrm{Fe}^{3+}\right)$.

The design and development of efficient and selective fluorescent sensors for metal ion detection is highly desirable due to their direct relation with the environment and water pollution ${ }^{35,36}$. Recently, GQDs are emerging as a novel type of fluorescence sensors due to their intriguing properties such as high solubility, low toxicity, chemical inertness, stable photoluminescence (PL), superior surface grafting, and so on ${ }^{37}$. In the context of strong luminescence properties, GQDs have been used to detect different analytes ${ }^{38-40}$. A number of sensors have been developed based on the PL quenching mechanism of GQDs ${ }^{41-43}$. Wang et al. was the first to report on $\mathrm{Fe}^{3+}$ ions detection by selective fluorescence quenching of $\mathrm{GQDs}^{43} . \mathrm{Fe}^{3+}$ is a very important metal ion present in both environmental and biological systems. $\mathrm{Fe}^{3+}$ ions can coordinate with different type of proteins present in biological systems. The concentration of $\mathrm{Fe}^{3+}$ ions plays an important role in the manipulation of Parkinson's disease (PD) since the accumulation of iron has been found at the affected neurons of PD patients ${ }^{44}$. Moreover, $\mathrm{Fe}^{3+}$ ions are among the substantial pollutants present in the environment and inducing water pollution ${ }^{45}$. In response to these reasons, the sensitive and selective detection of $\mathrm{Fe}^{3+}$ ions is urgently needed. Instrument-based detection and electrochemical sensing usually require complex preparations and are limited by their reproducibility and reliability ${ }^{45-47}$. Thus, the simple and sensitive detection by a luminescence-based approach make GQDs a very powerful tool for monitoring harmful metal ions in the environment.

In this study, GQDs have been prepared by a simple, yet efficient microwave treatment of carbon rich biochar obtained through the pyrolysis of biomass waste. The GQDs were synthesised in as short as a 30 min time span and exhibit excellent optical properties. A high product yield of $\sim 84 \%$ was achieved with increasing the reaction time or microwave power. Moreover, these GQDs were modified by simple hydrothermal treatment and displayed enhanced optical properties for the selective and sensitive detection of metal ions. In view of the PL properties of GQDs, a luminescence sensor was designed for $\mathrm{Fe}^{3+}$ sensing. It was shown that $\mathrm{Fe}^{3+}$ can be detected by GQDs leading to their fluorescence quenching. For the first time, this paper reports $\mathrm{Fe}^{3+}$ ion sensing using GQDs synthesised via microwave treatment of spent tea. Under the premise of employing a renewable cost-effective precursor, simple approach, acquiring high yield of GQDs and implementing in practical applications, our study lends further credence to the industrial potential of GQDs. Moreover, this procedure is generic and applicable to any other biomass waste which can yield carbon rich precursor by pyrolysis treatment.

\section{Results and discussion}

Synthesis and characterisation of GQDs. GQDs were successfully synthesised by oxidative cutting of a biomass-derived carbon rich precursor using nitric acid $\left(\mathrm{HNO}_{3}\right)$ as an oxidising agent via microwave heating of 15-180 min duration. Figure 1 shows a schematic illustration of the synthesis procedure for GQDs. The products obtained after each microwave treatment were examined by studying their optical properties.

In order to find out the best operating parameters a thorough study was carried out at a range of microwave powers (100-900 W) and processing times (15-180 min). The optical properties of the products were studied by recording their PL emission. Identical concentrations of $50 \mu \mathrm{g} \mathrm{mL}^{-1}$ were used for comparing the PL intensities of the GQDs prepared at different powers. The PL emission intensities of the products prepared at different microwave powers $(100,300,500$ and $900 \mathrm{~W}$ power) for a fixed duration of $120 \mathrm{~min}$, and at a range of treatment times $(15,30,60,120$ and $180 \mathrm{~min})$ at a fixed power of $500 \mathrm{~W}$, are compared in Fig. 2. The products obtained at $100 \mathrm{~W}$ power do not show significant optical emission, indicating that such a low microwave power could not induce cutting of carbon domains to generate GQDs. Therefore, the microwave power was further increased. It can be seen in Fig. 2a that the PL emission intensity increases with power up to $500 \mathrm{~W}$, while it declines with further increase in power to $900 \mathrm{~W}$. Similarly, the PL intensity increases with an increase in the treatment time up to $120 \mathrm{~min}$ and decreases thereafter (Fig. 2b). These results suggest that increase in power and process time intensify the cleavage of carbon domains into nanosized GQDs leading to enhanced optical emission, while increasing the power and time over a certain limit may cause the destruction of the surface and structure of GQDs resulting in diminished PL emission. These findings suggest that the intermediate values of $500 \mathrm{~W}$ power and 120 min process duration are the best conditions for the synthesis of high quality GQDs with excellent optical properties.

For further understanding, the optical properties of GQDs prepared at 500 and $900 \mathrm{~W}$ after 120 min of treatment (named as GQDs-500 and GQDs-900) were studied in detail by ultraviolet-visible (UV-Vis) absorption and PL emission spectroscopies. The UV-Vis absorption spectra of raw precursor, GQDs-500 and GQDs-900 are shown in Fig. 3a. The raw materials do not display notable UV absorbance, while both types of GQDs exhibit strong absorption in the UV range. The nature of both GQDs is similar and show absorption at $\sim 300 \mathrm{~nm}$ which indicates their identical absorbance states. The peaks observed at about $300 \mathrm{~nm}$ are due to $\mathrm{n} \rightarrow \pi^{*}$ transition of the carbonyl group $(\mathrm{C}=\mathrm{O})$, generally observed in graphene systems ${ }^{48}$. The non-bonding or ' $n$ ' electrons are the unpaired electrons on the oxygen of $\mathrm{C}=\mathrm{O}$. Therefore, $\mathrm{n}$ to $\pi^{*}$ transition takes place when one of the unpaired oxygen electrons is excited to the antibonding $\pi^{*}$ orbital $^{49}$. These results suggest that the UV absorbance in these GQDs is related to their surface oxygenated $(\mathrm{C}=\mathrm{O})$ states introduced during oxidative cutting with $\mathrm{HNO}_{3}$.

Since the absorbance in GQDs-500 and GQDs-900 is related to their identical absorbance states, a minor change in the intensity can be attributed to the difference in concentration of the absorbance states. Hence, the stronger UV absorbance of GQDs- 500 can be ascribed to their higher content of $\mathrm{C}=\mathrm{O}$ groups. A relatively 

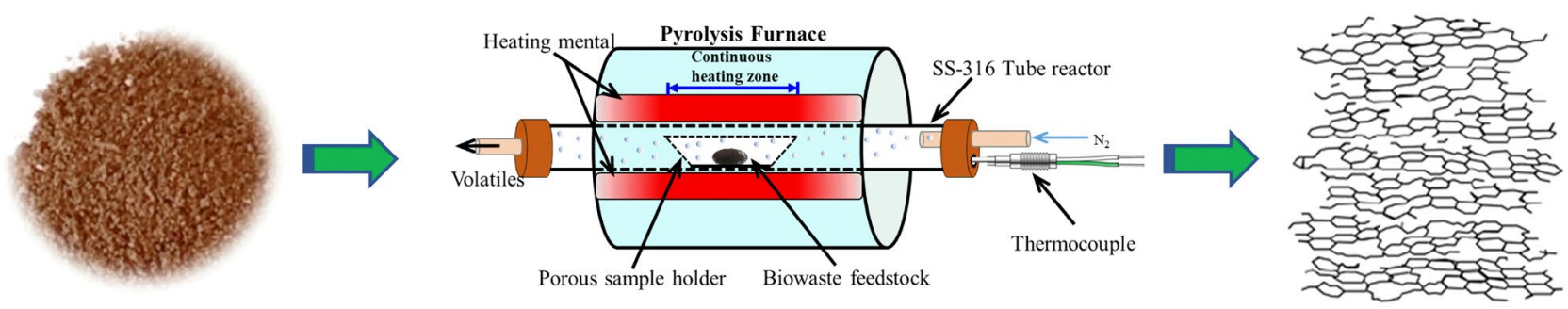

Spent tea

Carbon rich Precursor

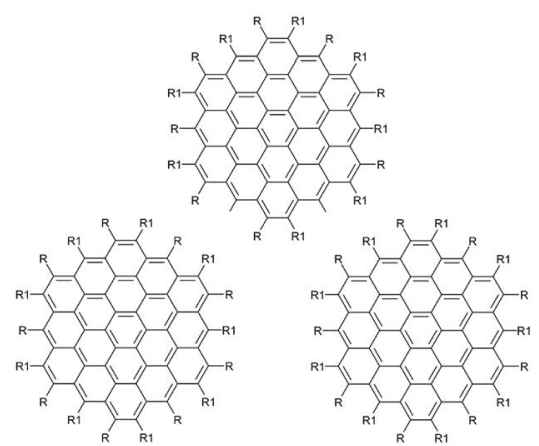

GQDs product
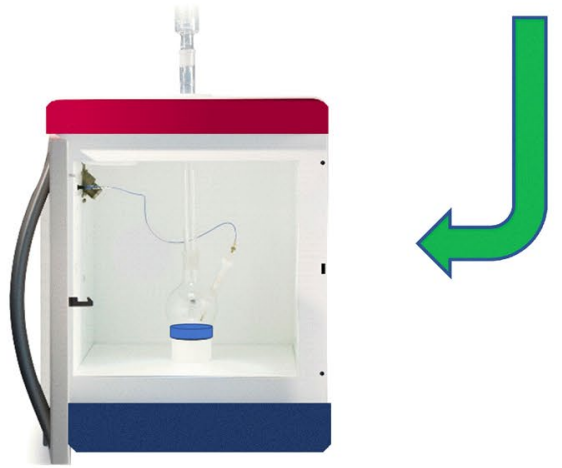

Figure 1. A schematic illustration of the synthesis procedure for graphene quantum dots (GQDs). Spent tea waste is subjected to pyrolysis treatment and carbon rich biochar is obtained, which is then microwave treated to produce GQDs with high yield. Figure was drawn using ChemDraw v16 (https://www.perkinelmer.com), Adobe Photoshop 2019 (https://www.adobe.com) and Microsoft PowerPoint 2016 (https://www.microsoft.com).
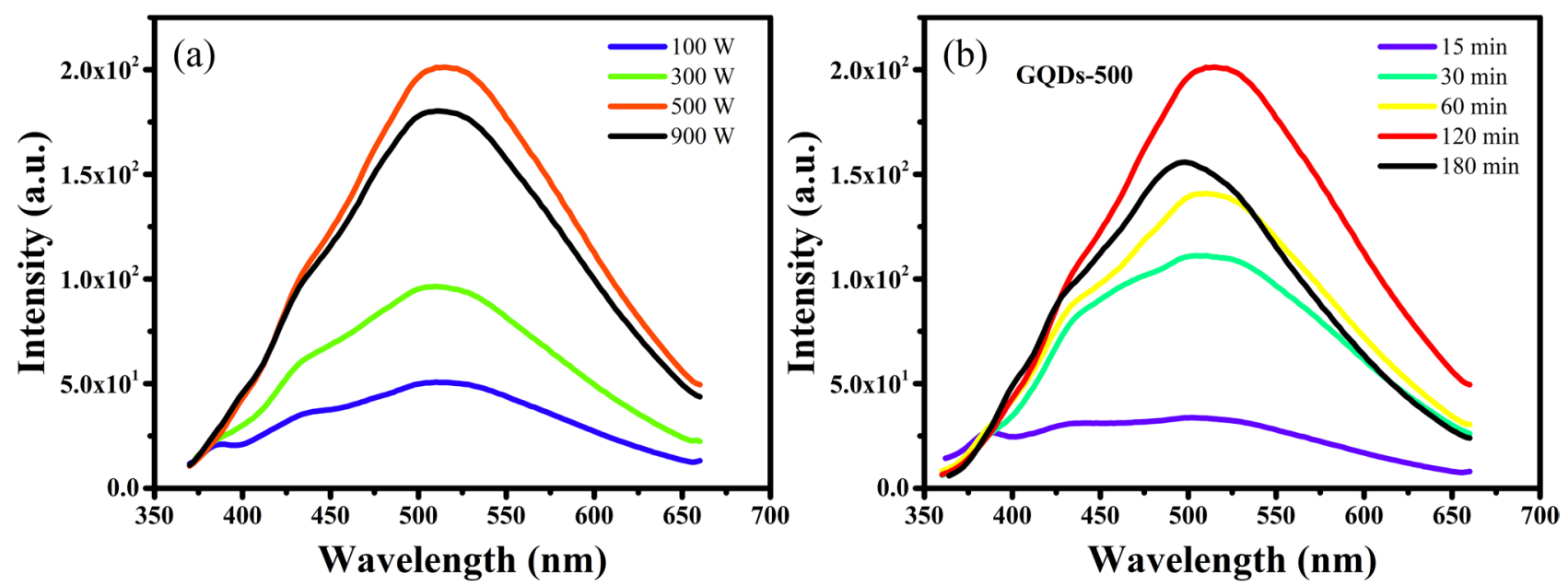

Figure 2. Comparison of the effect of (a) microwave power at a fixed duration of $120 \mathrm{~min}$ and (b) processing time at a fixed power of $500 \mathrm{~W}$, on the photoluminescence (PL) emission of GQDs at an excitation wavelength of $340 \mathrm{~nm}$. Figures were drawn using OriginPro 2018b (https://www.originlab.com).

week absorbance in GQDs-900 may be related to the destruction of the surface structure of GQDs-900 at high microwave power because the high temperature treatment removes the oxygen containing functional groups $\mathrm{s}^{50}$.

The optical bandgap of these GQDs was calculated based on the UV-Vis absorbance spectra using a Tauc plot $^{51}$. The curve was plotted for $(a h v)^{1 / \gamma}$ versus photon energy hv converted from the UV-Vis spectra, where, $a$ is optical absorption coefficient, $h$ is Planck's constant, $v$ is frequency of light, $A$ is a constant and $E_{g}$ is optical bandgap. The $\gamma$ factor depends on the nature of material and is equal to $1 / 2$ for direct bandgap materials and 2 for indirect bandgap materials $s^{52}$. A good linear fit was obtained while using $\gamma=1 / 2$ and no good fit was obtained when $\gamma=2$ was used, as shown in Fig. S1 (Supplementary Information). These results indicate a direct bandgap of GQDs studied here. The direct bandgap of both types of GQDs was estimated to be $\sim 5.12 \mathrm{eV}$ (see Supplementary Information for details). 

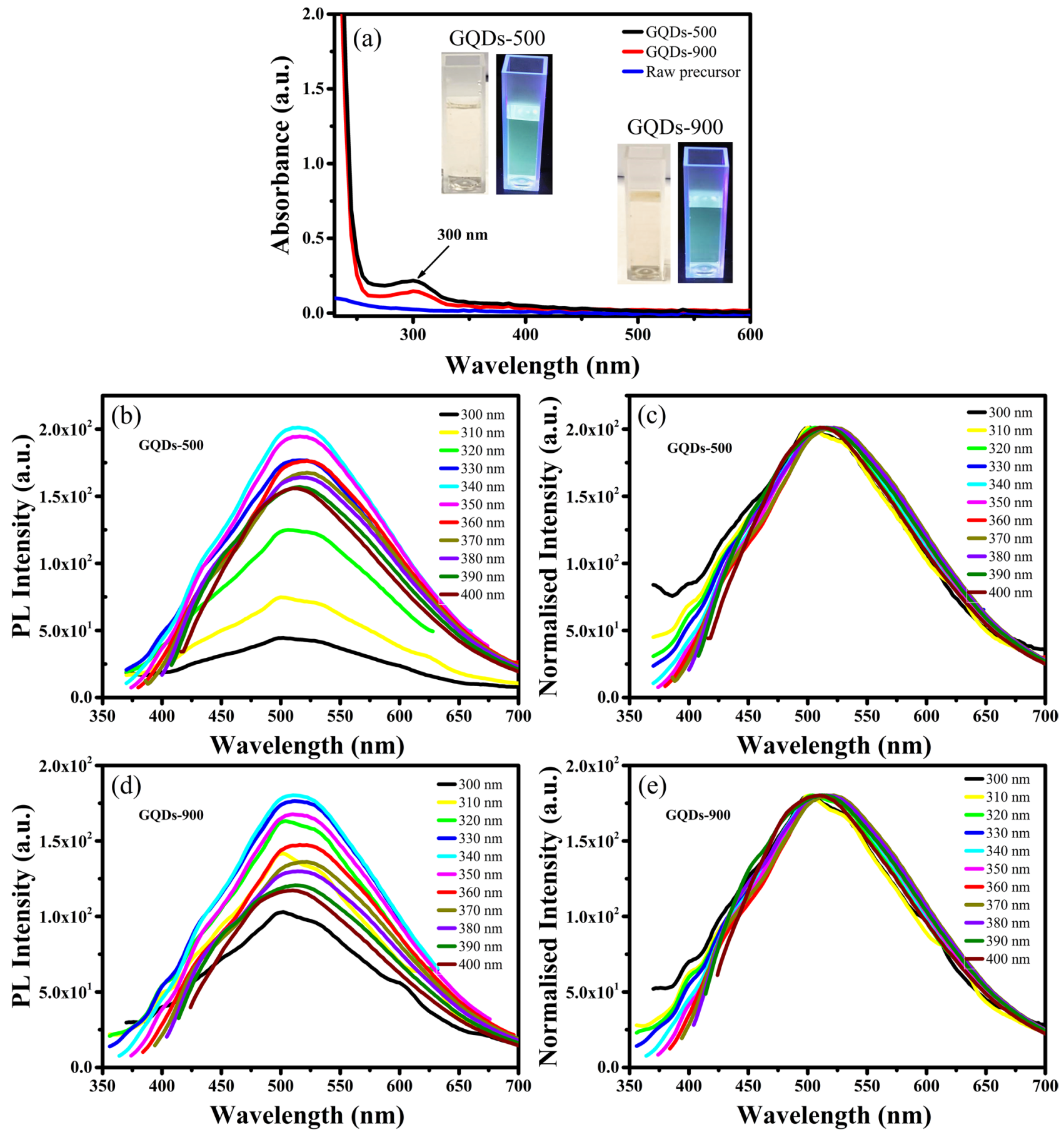

Figure 3. (a) Ultraviolet visible (UV-Vis) spectra of raw precursor, GQDs-500 and GQDs-900 showing a strong absorption of GQDs in the UV range (insets are the photographs of GQDs-500 and GQDs-900 under visible and $365 \mathrm{~nm}$ UV light). (b) PL spectra of GQDs-500 at different excitation wavelengths and (c) their normalised PL spectra exhibiting excitation independent emission. (d) PL spectra of GQDs-900 and (e) their normalised PL spectra showing no shift in PL peak position. Inset images in (a) were taken using iPhone Xs Max (https://www. apple.com) and graphics in (a)-(e) were plotted using OriginPro 2018b (https://www.originlab.com).

Insets in Fig. 3a show photographs of the GQDs-500 and GQDs-900 solutions under visible light and $365 \mathrm{~nm}$ UV light. Both types of GQDs display a light-yellow colour under visible light while a green fluorescence is observed under UV light. The optical emission of GQDs was explored by carrying out a detailed study of photoluminescence emission at various excitation wavelengths. Figure 3b-e shows the PL spectra of GQDs-500 and GQDs-900 at various excitation wavelengths. Both types of GQDs exhibit excitation independent PL emission at a range of excitation wavelengths from 300 to $400 \mathrm{~nm}$. When excited by $300 \mathrm{~nm}$ excitation wavelength, the GQDs-500 display a weak PL emission with a peak at $~ 514 \mathrm{~nm}$. The PL intensity of GQDs-500 increases gradually with increase in the excitation wavelength and exhibit a strongest emission at $340 \mathrm{~nm}$. The PL intensity decreases 


\begin{tabular}{|l|l|l|}
\hline Microwave power (W) & Microwave duration (min) & Product yield (wt.\%) \\
\hline \multirow{5}{*}{500} & 15 & 5 \\
\cline { 2 - 3 } & 30 & 20 \\
\cline { 2 - 3 } & 60 & 25 \\
\cline { 2 - 3 } & 120 & 73 \\
\cline { 2 - 3 } & 180 & 84.5 \\
\hline \multirow{4}{*}{900} & 15 & 10 \\
\cline { 2 - 3 } & 30 & 33 \\
\cline { 2 - 3 } & 60 & 80 \\
\cline { 2 - 3 } & 120 & 84 \\
\hline
\end{tabular}

Table 1. Values of the product yield obtained at different microwave powers and time periods.

thereafter and gradually stabilises at about $390 \mathrm{~nm}$. Therefore, the PL intensities at 390 and $400 \mathrm{~nm}$ excitation are almost the same. Similarly, GQDs-900 also show PL emission having a peak at $514 \mathrm{~nm}$ when excited by $300 \mathrm{~nm}$ light. The PL intensity of GQDs-900 also varies with change in the excitation wavelength and shows the strongest emission at $340 \mathrm{~nm}$ excitation. These results suggest that both types of these GQDs are similar in nature.

Most of the carbon-based QDs exhibit excitation dependent PL emission ${ }^{1,8,13,53-56}$. Their PL emission peak positions change with the change in excitation wavelength. However, it is interesting to note that GQDs prepared through the present approach demonstrate an excitation independent emission. Though the PL intensity changes with a change in excitation wavelength for both types of GQDs, however, they exhibit excitation independent PL emission. When the excitation wavelength is changed from 300 to $400 \mathrm{~nm}$, the intensity of PL emission increases to reach a maximum at $340 \mathrm{~nm}$ excitation, and decreases thereafter, however the peak position does not shift and remains fixed at $\sim 514 \mathrm{~nm}$. In order to check the peak shifting, the PL spectra were normalised to the maximum of the strongest emission (340 nm excitation). The normalised PL spectra in Fig. 3c,e show no shifting in peak positions with change in excitation wavelength and this further confirms the independency of PL emission from the excitation wavelength. This characteristic of the present GQDs is different from other carbon based QDs ${ }^{18,24,56,57}$. Therefore, it requires more in-depth understanding of their formation mechanism.

Several mechanisms have been proposed to explain the origin of PL emission of carbon QDs. The most common ones involve oxygenated functional groups, quantum size, zigzag edge sites, defect effects and recombination of electron-hole pairs ${ }^{58-62}$. Most of the GQDs produced so far exhibit excitation dependent emission which is generally attributed to surface emissive traps and defect states ${ }^{63}$. However, the zigzag edge sites, quantum size and recombination of electron-hole pairs are regarded as single emission fluorescence centres ${ }^{64,65}$ and generate excitation independent emission ${ }^{66}$. It was reported ${ }^{63,67}$ that the PL emission from GQDs at $500-530 \mathrm{~nm}$ is mainly attributed to the conjugated carbon skeleton containing zigzag edge sites. Thus, the excitation independent emission of the present GQDs at $514 \mathrm{~nm}$ may be attributed to the zigzag sites ${ }^{66}$ and $n-\pi^{*}$ transition of $\mathrm{C}=\mathrm{O}$ groups, consistent with their absorbance spectra. Therefore, it is suggested that the present GQDs prepared via microwave irradiation do not have considerable emissive traps or defect states and exhibit PL due to single emission fluorescence centre ${ }^{64,66}$. Since the prepared GQDs displays this unique optical characteristic, this makes them ideal for numerous applications such as lasing, display technologies, multi-target bioimaging and encryption ${ }^{68}$.

The effect of microwave power and treatment time on the product yield was systematically studied. The product yield was recorded for each treatment based on the biochar precursor and the results are summarised in Table 1. The product yield of both types of GQDs increases with increasing reaction time.

The highest product yield of around $84 \%$ is achieved for a reaction time of $180 \mathrm{~min}$ at microwave power of $500 \mathrm{~W}$ or $120 \mathrm{~min}$ at $900 \mathrm{~W}$ power. These results suggest that higher microwave power could induce rapid cutting of carbon domains into GQDs. To the best of the authors' knowledge, these are the highest values of product yield reported to date for the synthesis of GQDs from biomass waste. Table 2 summarises the product yield of GQDs derived from various biomass-based precursors studied previously.

The quantum yields of GQDs were calculated by comparing their integrated PL intensities and absorbance values with that of quinine sulfate. Quinine sulfate was dissolved in $0.1 \mathrm{M} \mathrm{H}_{2} \mathrm{SO}_{4}$ having a quantum yield of 0.54. As-prepared GQDs-500 and GQDs-900 were dissolved in water and concentrations were adjusted to obtain an absorbance value of less than 0.1 in order to avoid re-absorption effects. A $10 \mathrm{~mm}$ cuvette was used and slit widths were fixed at $5.0 \mathrm{~nm}$ for both excitation and emission. The following equation was used to calculate the quantum yield ${ }^{79}$ :

$$
\phi=\phi_{\mathrm{r}} \frac{\mathrm{I}(\mathrm{Ar}) \mathrm{n}^{2}}{\mathrm{I}_{\mathrm{r}}(\mathrm{A}) \mathrm{n}_{\mathrm{r}}^{2}}
$$

where, $\phi$ and $\phi_{\mathrm{r}}$ are the quantum yields of the sample and standard reference, I and $\mathrm{I}_{\mathrm{r}}$ are the integrated PL intensities of the sample and reference, Ar and $A$ are the absorbance values and $n_{r}$ and $n$ are the refractive indices of the reference and required sample, respectively. The quantum yields of as-prepared GQDs-500 and GQDs-900 obtained after $120 \mathrm{~min}$ of treatment were calculated to be 0.09 and 0.07 , respectively.

Structural studies of GQDs were performed by transmission electron microscopy (TEM). TEM images along with particle size distribution of GQDs-500 and GQDs-900 obtained after 120 min of treatment are presented in 


\begin{tabular}{|l|l|l|l|l|}
\hline Precursor & Product & Synthesis approach & Product yield (wt\%) & Refs. \\
\hline Spent tea derived carbon & GQDs & Microwave treatment & $5-84.5$ & Present work \\
\hline Plant leaf extract & N-GQDs & Hydrothermal treatment & 25.2 & 69 \\
\hline Paper lignin & GQDs & Pyrolysis & $8.74-18.02$ & 70 \\
\hline Neem leaves & GQDs & Pyrolysis & - & 39 \\
\hline Rice-husk carbon & GQDs & Pyrolysis + Hydrothermal treatment & 15 & 71 \\
\hline Coffee grounds & GQDs & Hydrothermal treatment & 33 & 72 \\
\hline Durian & S-GQDs & Hydrothermal treatment & $6.8 \%$ & 23 \\
\hline Alkali lignin & GQDs & Hydrothermal treatment & 21 & 73 \\
\hline Garlic & CQDs & Hydrothermal treatment & - & 74 \\
\hline Fresh tomato & Carbon dots & Microwave pyrolysis & - & 75 \\
\hline Coconut shells & CQDs & Hydrothermal treatment & - & 76 \\
\hline Chitosan & CQDs & Hydrothermal treatment & 10 & 77 \\
\hline Taxus leaves & Carbon dots & Hydrothermal treatment & 1 & 78 \\
\hline
\end{tabular}

Table 2. Comparison of the product yield of quantum dots (QDs) derived from various biomass-based precursors.

(a)
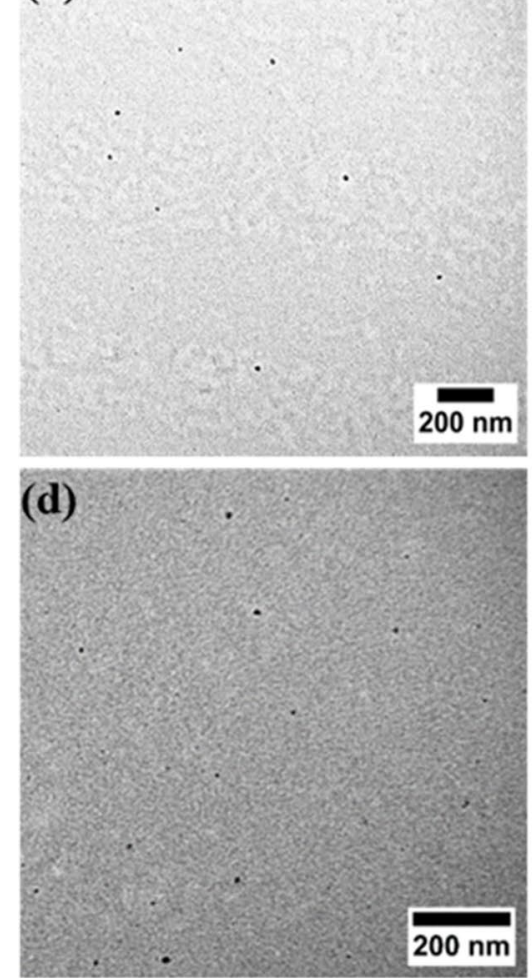

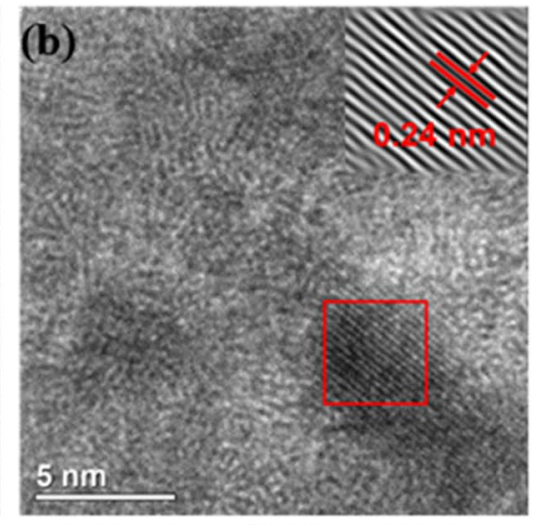

\section{(e)}

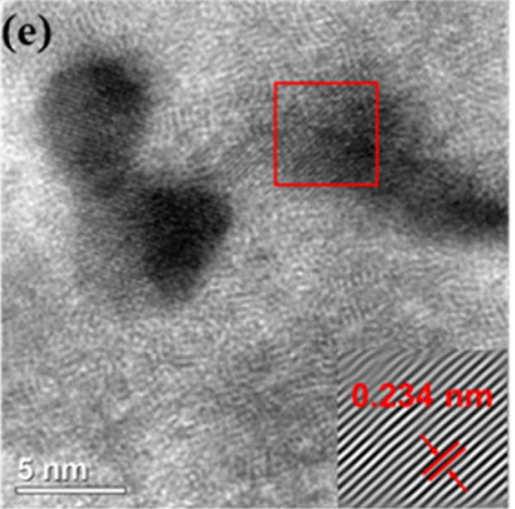

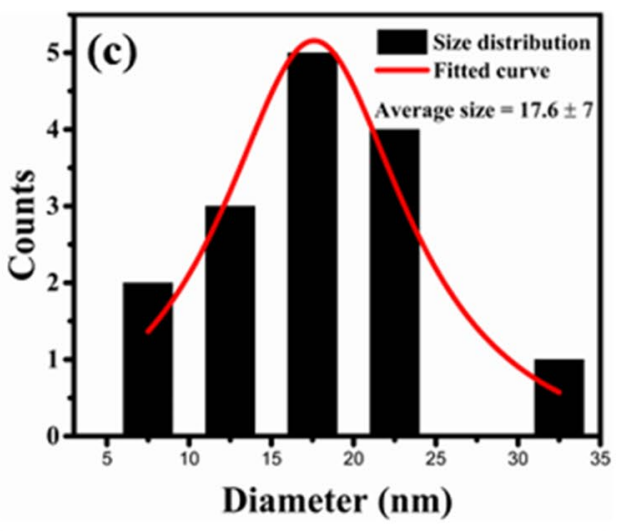

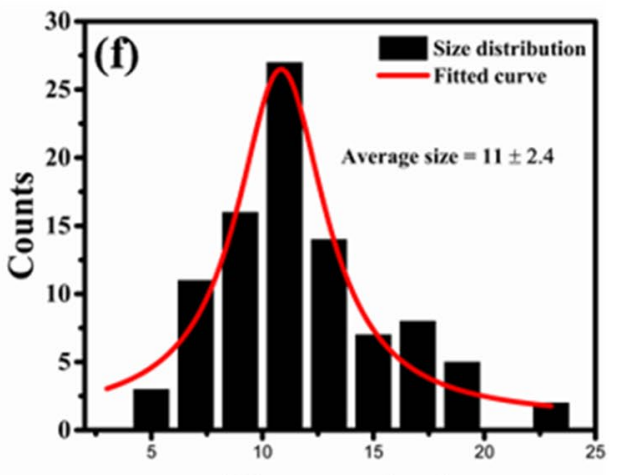

Diameter (nm)

Figure 4. (a) TEM image, (b) HRTEM image and (c) respective particle size distribution of GQDs-500. (d) TEM, (e) HRTEM and (f) size distribution measurements of GQDs-900. Both types of GQDs were obtained after 120 min of treatment. Images in (a), (b), (d) and (e) were processed using DigitalMicrograph 3.40.2804.0 (https://www.gatan.com) and plotted using ImageJ 1.8.0_112 (https://imagej.nih.gov/ij/). Figures (c) and (e) were plotted using OriginPro 2018b (https://www.originlab.com).

Fig. 4. The TEM results verify the synthesis of nanosized GQDs by microwave treatment. The 'as prepared' GQDs are well dispersed and possess almost uniform shape. Careful size measurements using the software 'ImageJ' show that the GQDs-500 are in the size range of $\sim 6$ to $\sim 34 \mathrm{~nm}$ (Fig. $4 \mathrm{c}$ ). The average size was calculated to be $\sim 17.6 \mathrm{~nm}$. The high resolution TEM (HRTEM) image (Fig. 4b) shows the crystalline structure of GQDs and a lattice spacing of $\sim 0.24 \mathrm{~nm}$ corresponding to (1120) plane of graphene ${ }^{71}$. An average size below $100 \mathrm{~nm}$, graphene structure and strong optical properties indicate the product obtained is typically GQD 6 . On the other hand, the GQDs900 exhibit uneven shape with a size range of 4 to $24 \mathrm{~nm}$, with an average size of $11 \mathrm{~nm}$ (Fig. $4 \mathrm{~d}-\mathrm{f}$ ). The size measurements show that the overall size of GQDs-900 prepared at $900 \mathrm{~W}$ is smaller than the size of GQDs-500 

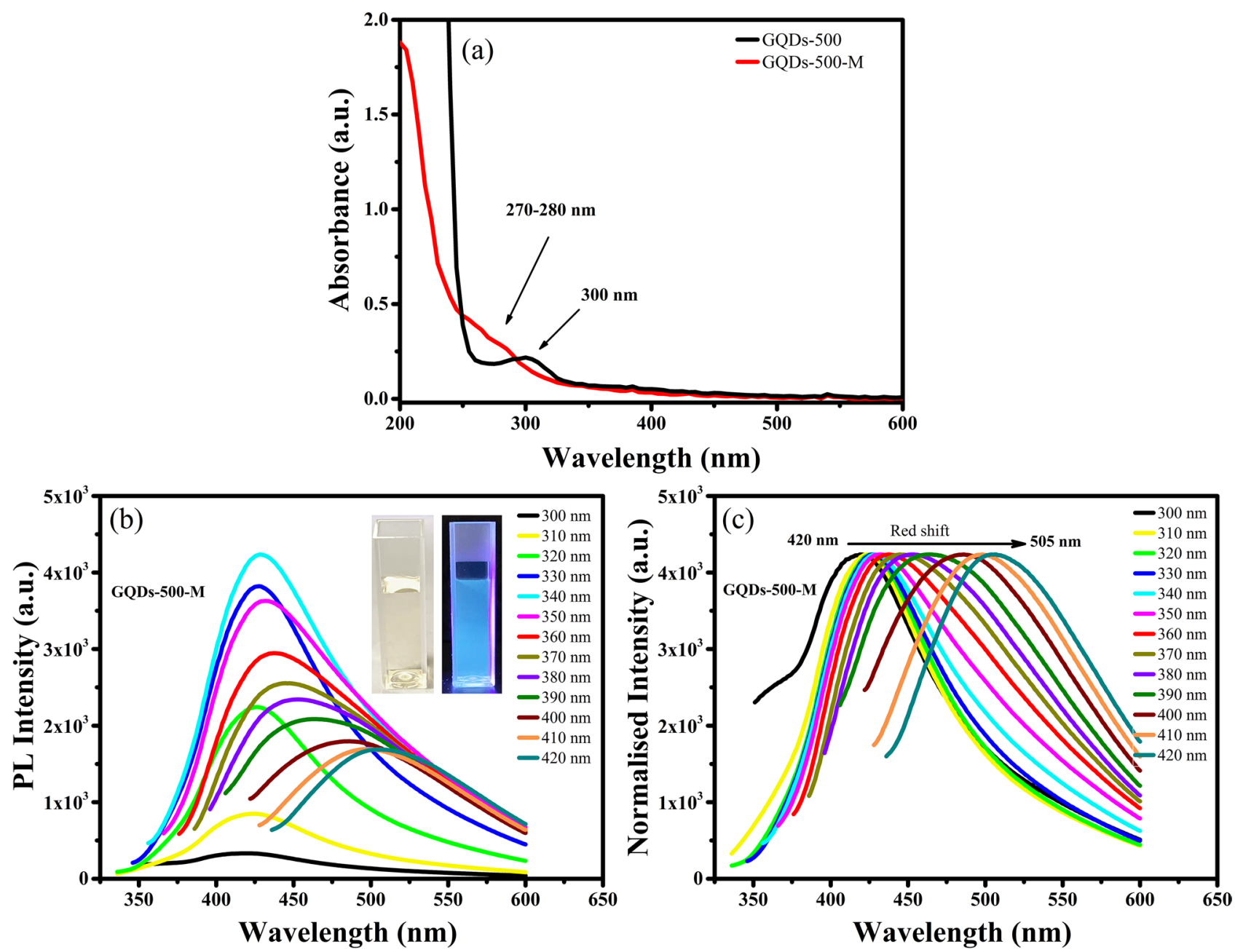

Figure 5. (a) UV-Vis spectra of GQDs-500 and GQDs-500-M showing a strong absorption at 300 and 270$280 \mathrm{~nm}$ range, respectively. (b) The PL spectra of GQDs-500-M at a range of excitation wavelengths (insets are the photographs of GQDs-500-M solution under visible and $365 \mathrm{~nm}$ UV light) and (c) normalised PL spectra showing a red shift in PL emission. Figures (a)-(c) were plotted using OriginPro 2018b (https://www.originlab. com) and inset photographs in (b) were taken using iPhone Xs Max (https://www.apple.com).

prepared at $500 \mathrm{~W}$. This suggests that higher power will break the carbon skeleton more significantly and generate relatively smaller particles. The HRTEM image of GQDs-900 in Fig. 4e displays a lattice spacing of $0.234 \mathrm{~nm}$ related to $(1120)$ plane of graphene $(0.24 \mathrm{~nm})^{71}$. These results indicate the graphene structure of both type of GQDs prepared at 500 and $900 \mathrm{~W}$ powers. However, higher power had a detrimental effect on the surface and structure of GQDs-900 as noticed in TEM/HRTEM images and variation in the lattice spacing parameter. This structural destruction may partially contribute to a decreased PL emission in GQDs-900. Owing to the superior optical properties and uniform shape and size, GQDs-500 were considered for the further study.

A number of techniques have been implemented to enhance the optical properties and quantum yield of GQDs such as heteroatom doping, surface-functionalisation, size modification, etc ${ }^{1,3}$. Hydrothermal treatment is a simple yet efficient approach for reducing the size of GQDs ${ }^{18}$. Hence, this approach was adopted to modify the size of GQDs-500 and study its effect on the optical properties. This process provides an insight into the enhancement of optical properties of GQDs prepared by different approaches.

Property and structural studies of modified GQDs. The GQDs-500 were further modified by hydrothermal treatment and denoted as "GQDs-500-M", where ' $M$ ' indicates the modified version. The GQDs-500-M were dissolved in water with a concentration of $50 \mu \mathrm{g} \mathrm{mL}^{-1}$ and their optical properties were comprehensively studied by UV-Vis and PL spectroscopies. The UV-Vis spectra of GQDs-500 and GQDs-500-M are displayed in Fig. 5a. The results indicate a strong UV absorption of GQDs-500-M, consistent with the observations of their unmodified forms. The absorption peaks are observed at $\sim 270-280 \mathrm{~nm}$, which are attributed to $\pi-\pi^{*}$ transition of $\mathrm{C}=\mathrm{C}$ in aromatics ${ }^{80}$, indicating graphene structure of the GQDs. The optical bandgap of GQDs-500-M was calculated using Tauc plot derived from the absorbance spectrum and determined to be $\sim .4 \mathrm{eV}$ (see Fig. S1, Supplementary Information). It is worth noting that the bandgap is increased after modification. Moreover, the 
absorbance spectrum of GQDs-500-M shows a blue shift as compared to their unmodified counterparts. These results indicate a change in optical properties of GQDs after modification.

In order to get further insight into the optical properties, the GQDs-500-M were characterised by PL spectroscopy. The PL emission spectra of GQDs-500-M are presented in Fig. 5b. Inset in Fig. 5b shows photographs of the GQDs-500-M solutions under visible light and $365 \mathrm{~nm}$ UV light. The GQDs-500-M solution displays a light-yellow transparent colour under visible light, while a strong blue fluorescence is observed when illuminated by $365 \mathrm{~nm}$ UV light. The optical emission of GQDs-500-M was recorded by exposing to a range of excitation wavelengths from 300 to $400 \mathrm{~nm}$ and measuring the corresponding emission spectra. When excited by $300 \mathrm{~nm}$ laser, the GQDs-500-M show emission at $\sim 420 \mathrm{~nm}$. The emission wavelength is higher than the excitation, which is known as Stokes shift. The fluorescence intensity increased with increasing the excitation wavelength up to an optimum level and then diminished. The strongest emission was observed at $\sim 430 \mathrm{~nm}$ when excited by $340 \mathrm{~nm}$ laser. Importantly, the emission peak position changes with the change in excitation wavelength (Fig. 5c). Consequently, the GQDs-500-M exhibit broad emission spectra from a shorter wavelength (blue) of $400 \mathrm{~nm}$ to a longer wavelength (near-red) of $550 \mathrm{~nm}$. These results imply the excitation dependent PL emission characteristic of GQDs-500-M. This property suggests the use of these GQDs in multicolour imaging applications (such as bioimaging).

In contrast with unmodified GQDs, the common mechanism suggested for excitation dependent emission in GQDs-500-M is quantum confinement effect, surface emissive traps or different types of functional groups introducing various emissive states which are excited by photons of different energies, resulting in excitation dependent PL emission ${ }^{4,30,39}$. The quantum yield of GQDs-500-M was calculated using Eq. (1) and found to be 0.23 . The quantum yield of GQDs- 500 was significantly improved after modification, thus highlighting the substantial advantages of improving optical properties using simple hydrothermal treatment.

The structure and size distribution of modified GQDs were studied by TEM analysis. Low and high magnification TEM images of the GQDs-500-M are presented in Fig. 6, which show a uniform dispersion of modified GQDs. The images show a drastic decrease in the size of modified GQDs as compared to their unmodified form (GQDs-500). The high magnification TEM image (Fig. 6b) further shows that the particles are well dispersed and exist in a narrow size range. The size distribution of these GQDs was calculated using ImageJ and the results are presented in Fig. 6d. The results show that the size of GQDs-500-M falls in the range of 0.5 to $3.5 \mathrm{~nm}$, with an average of $1.6 \pm 0.55 \mathrm{~nm}$. The results demonstrate a very narrow size distribution. The high resolution TEM image (Fig. 6c) reveals the crystalline structure of modified GQDs. The lattice spacing were calculated to be $\sim 0.24 \mathrm{~nm}$ corresponding to (1120) plane of graphene $\mathrm{e}^{71}$.

The structural characterisation of GQDs was performed by Raman and Fourier transform infrared (FTIR) analysis. The Raman spectrum (Fig. 7) of GQDs-500-M consists of two distinctive bands at about 1356 and $1569 \mathrm{~cm}^{-1}$, known as disorder (D) band and crystalline (G) band, respectively. The D and $\mathrm{G}$ bands in the range of $1200-1800 \mathrm{~cm}^{-1}$ are indicative of a carbonaceous structure ${ }^{81,82}$. The $\mathrm{D}$ band is related to the defect mediated zone-edge (near K-point) phonons that shows the defects, edges and disorder in the carbon lattice.

The $\mathrm{G}$ band is associated with the in-plane vibration of $\mathrm{sp}^{2}$ carbon atoms and usually indicates the crystallinity of the carbon ${ }^{83}$. Moreover, the intensity ratio of $\mathrm{D}$ band and $\mathrm{G}$ band $\left(\mathrm{I}_{\mathrm{D}} / \mathrm{I}_{\mathrm{G}}\right)$ shows the density of disorder in the structure of carbon ${ }^{83,84}$. The $\mathrm{I}_{\mathrm{D}} / \mathrm{I}_{\mathrm{G}}$ value of GQDs- $500-\mathrm{M}$ is $\sim 0.98$, which show their defective structure, possibly arising from the edge states at the periphery of GQDs ${ }^{81}$. These results are in good agreement with previously reported GQDs ${ }^{53,54,81}$

Figure 7b shows the FTIR spectrum of GQDs-500-M. The spectrum shows a broad band at $\sim 3300 \mathrm{~cm}^{-1}$ corresponding to the stretching vibrations of hydroxyl $(\mathrm{O}-\mathrm{H})$ and amine $(\mathrm{N}-\mathrm{H})$ groups. The peaks at $\sim 1630$ and $\sim 1560 \mathrm{~cm}^{-1}$ are related to the stretching vibrations of $\mathrm{C}=\mathrm{O}$ in carbonyl groups and stretching vibrations of $\mathrm{C}=\mathrm{C}$ in aromatic groups, respectively. The multiple peaks at $1350-1500 \mathrm{~cm}^{-1}$ are attributed to the bending vibrations of $\mathrm{C}-\mathrm{C} / \mathrm{C}-\mathrm{H}$ in alkanes. The bands at $1100-1250 \mathrm{~cm}^{-1}$ are due to stretching vibrations of $\mathrm{C}-\mathrm{N}$ groups in amines and $\mathrm{C}-\mathrm{O}$ groups in ethers, esters, carboxylic acids and alcohols. A broad band at $\sim 1000 \mathrm{~cm}^{-1} \mathrm{cor}^{-}$ responds to the $\mathrm{C}-\mathrm{O}$ stretching of ether groups ${ }^{85,86}$. These results suggest large amounts of oxygen and nitrogen functional groups on the surface of GQDs-500-M. These functional groups make them hydrophilic in nature and render them highly soluble in water.

The surface functionalisation and elemental composition of the GQDs-500-M were further confirmed by $\mathrm{X}$-ray photoelectron spectroscopy (XPS). The survey XPS spectrum shows three major peaks at 285.08, 400.08 and $532.08 \mathrm{eV}$, which are assigned to C1s, N1s and O1s, respectively (Fig. 7c). Another small peak at $347.08 \mathrm{eV}$ associated with $\mathrm{Ca} 2 \mathrm{p}$ is also observed. The XPS elemental analysis reveals that the GQDs-500-M are mainly composed of C (56.45 at.\%), O (36.73 at.\%) and N (4.76 at.\%), with a limited content of Ca from the feedstock. Ash content in the biomass derived biochar precursor is the origin of calcium and it is present as a $\mathrm{CaCO}_{3}$-like species $^{87}$.

The high resolution $\mathrm{C} 1 \mathrm{~s}$ spectrum (Fig. $7 \mathrm{~d}$ ) can be deconvoluted into three peaks at $\sim 284.9, \sim 286.3$, and $\sim 288.4 \mathrm{eV}$, corresponding to $\mathrm{C}-\mathrm{C} / \mathrm{C}=\mathrm{C}, \mathrm{C}-\mathrm{O}-\mathrm{C}$ and $\mathrm{O}-\mathrm{C}=\mathrm{O}$ groups, respectively ${ }^{60}$. The $\mathrm{N} 1 \mathrm{~s}$ spectrum (Fig. 7e) can be fitted into three peaks at $\sim 399.89, \sim 402.01$ and $406.05 \mathrm{eV}$, which can be attributed to the pyridinic/pyrrolic nitrogen $(\mathrm{C}-\mathrm{N} / \mathrm{N}-\mathrm{H})$, graphitic nitrogen $\left(\mathrm{N}-(\mathrm{C})_{3}\right)$ and nitro $(\mathrm{N}-\mathrm{O})$ species, respectively ${ }^{78,88}$. The $\mathrm{O} 1 \mathrm{~s} \mathrm{spectrum} \mathrm{(Fig.} \mathrm{7f)} \mathrm{consists} \mathrm{of} \mathrm{two} \mathrm{major} \mathrm{peaks} \mathrm{at} 531.58$ and $533.08 \mathrm{eV}$, which can be ascribed to $\mathrm{O}=\mathrm{C}$ and $\mathrm{O}-\mathrm{C}$, respectively ${ }^{60,89}$. These results suggest that the hydrothermal treatment not only modified the size of GQDs but also incorporated extensive content of oxygen and nitrogen functional groups in the matrix of modified GQDs, making them highly soluble in water with good stability. Moreover, no detectable changes were observed in the GQDs-500-M after storing for two months which further verifies their good stability. 

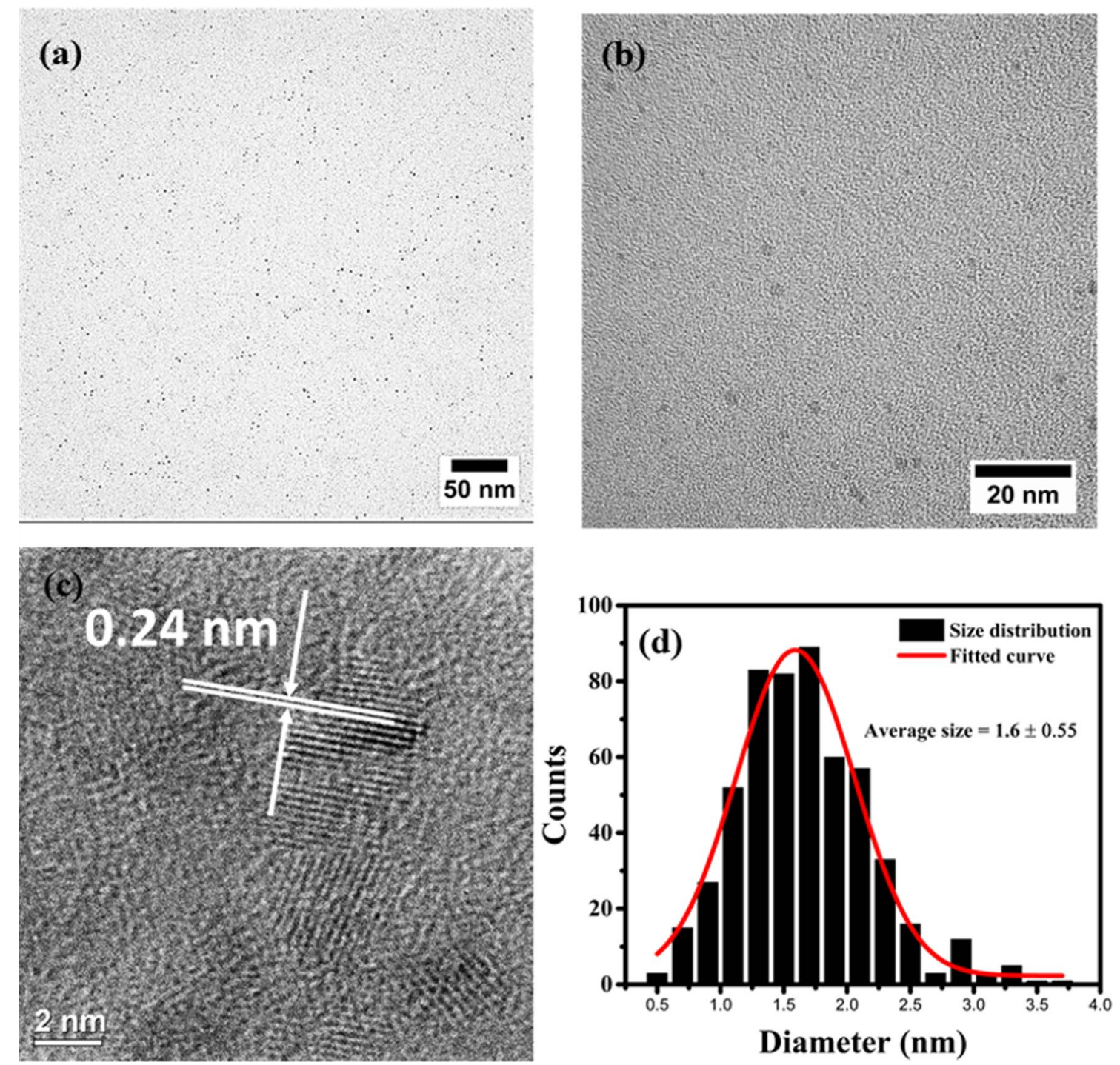

Figure 6. (a) Low magnification TEM image, (b) high magnification TEM image (c) high resolution TEM (HRTEM) image and (d) corresponding size distribution of the GQDs-500-M. Images in (a), (b) and (c) were processed using DigitalMicrograph 3.40.2804.0 (https://www.gatan.com) and plotted using ImageJ 1.8.0_112 (https://imagej.nih.gov/ij/). Figure (d) was plotted using OriginPro 2018b (https://www.originlab.com).

Application as a PL sensor. The as-synthesised GQDs-500-M exhibit strong PL emission and specific oxygenated surface functionalisation. The presence of these functional groups result in the hydrophilicity of the GQDs leading to good solubility in water ${ }^{55,90,91}$. Owing to the presence of these functional groups and strong emission, these GQDs are expected to be an ideal candidate for fluorescence sensing.

Among the various heavy metal ions, the detection and removal of $\mathrm{Fe}^{3+}$ ions has always attracted extensive attention ${ }^{53,92,93}$. Generally, iron plays a key role in human life and its deficiency requires considerable attention among the global micronutrient deficiencies. Moreover, the iron percentage has to be monitored and balanced in biological and environmental systems ${ }^{94}$. To achieve this, numerous researchers employed the quenching phenomenon of carbon QDs by the addition of $\mathrm{Fe}^{3+4,53,95}$. The quenching percentage is related to the concentration of $\mathrm{Fe}^{3+}$.

In this study, the selective sensing of $\mathrm{Fe}^{3+}$ was performed by $50 \mu \mathrm{g} \mathrm{mL}^{-1} \mathrm{GQDs}-500-\mathrm{M}$ solution using $100 \mu \mathrm{M}$ concentration of fifteen different metal ions: $\mathrm{Ag}^{1+}, \mathrm{Al}^{3+}, \mathrm{Ca}^{2+}, \mathrm{Co}^{2+}, \mathrm{Cr}^{3+}, \mathrm{Cu}^{2+}, \mathrm{Mg}^{2+}, \mathrm{Mn}^{2+}, \mathrm{Mo}^{2+}, \mathrm{Fe}^{3+}, \mathrm{Sr}^{1+}, \mathrm{Zn}^{2+}$, $\mathrm{Na}^{1+}, \mathrm{Li}^{1+}$ and $\mathrm{Fe}^{2+}$. As discussed earlier, the GQDs-500-M exhibit a strong PL emission when excited by a wavelength of $340 \mathrm{~nm}$. Therefore, this wavelength was selected to monitor the effect of metal ions on the PL intensity. The PL intensities of GQDs-500-M were recorded with the addition of different metal ions. As shown in Fig. 8a, comparing with the control (blank) sample, the PL of GQDs was remarkably quenched only by $\mathrm{Fe}^{3+}$ ions among fifteen different metal ions. These results suggest the highly selective detection of $\mathrm{Fe}^{3+}$ by the GQDs-500-M.

The mechanism of PL quenching is related to the strong affinity of $\mathrm{Fe}^{3+}$ ions towards the hydroxyl/carboxyl groups of GQDs-500-M, leading to a stable complex ${ }^{13}$. PL quenching occurs when the excited electron in GQDs partially transfers to the $\mathrm{d}$ orbital of $\mathrm{Fe}^{3+}$ instead of undergoing radiative relaxation ${ }^{13}$. The affinity (coordination) of numerous metal ions toward GQDs-500-M was evaluated systematically under similar experimental 

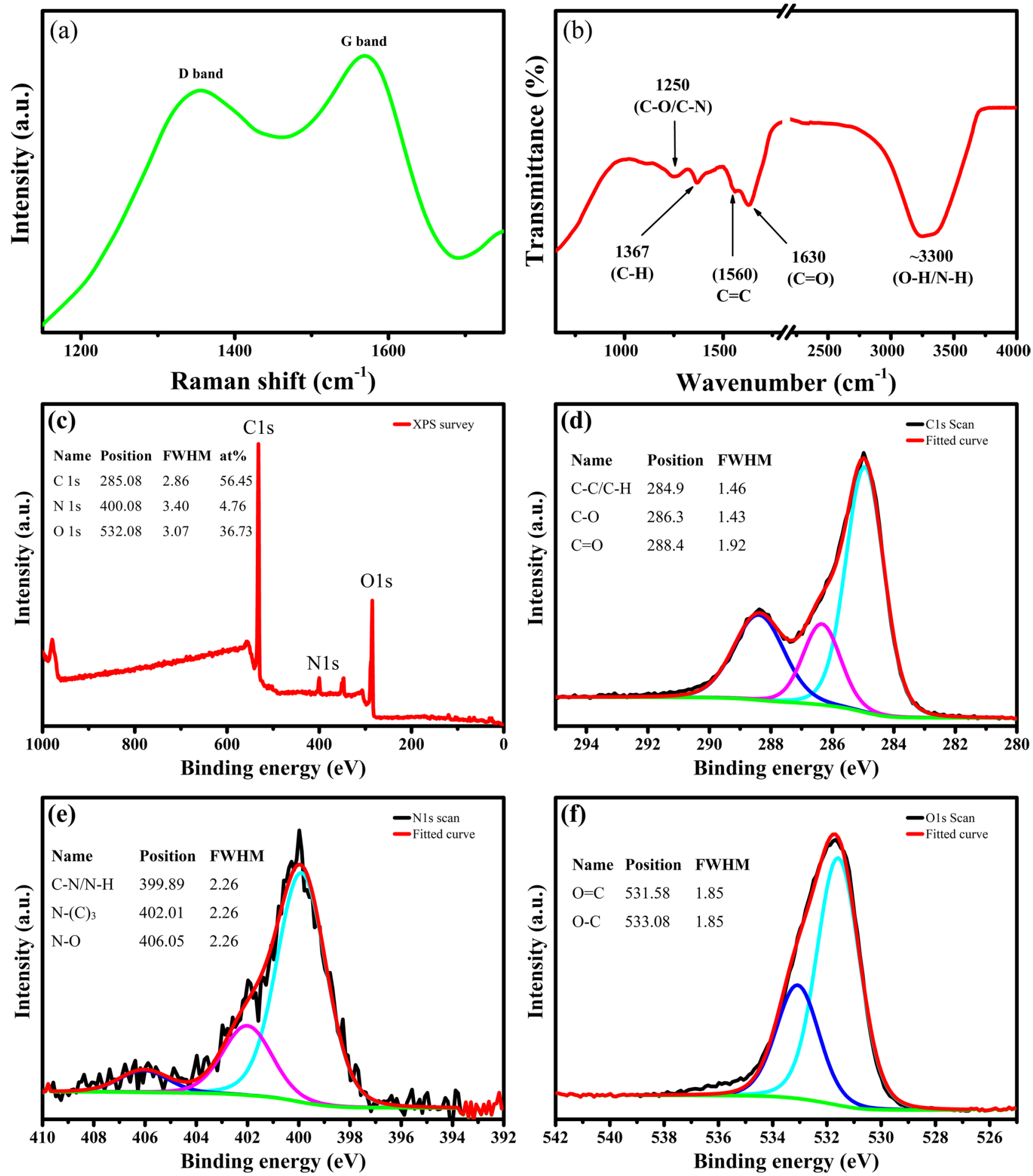

Figure 7. (a) Raman and (b) FTIR spectra of GQDs-500-M displaying the oxygen and nitrogen functional groups. (c) X-ray photoelectron spectroscopy (XPS) survey spectrum, (d) C1s, (e) N1s and (f) O1s high resolution spectra of GQDs-500-M, showing extensive number of oxygen and nitrogen functionalities. Drawings were plotted using OriginPro 2018b (https://www.originlab.com).

conditions. Figure $8 \mathrm{~b}$ shows that the $\mathrm{Fe}^{3+}$ ions exhibit strongest affinity towards GQDs-500-M among fifteen different metal ions, suggesting its potential for selective sensing by GQDs-500-M.

Further study was carried out to investigate the effect of $\mathrm{Fe}^{3+}$ concentration on the fluorescence intensity of GQDs. The PL spectra of GQDs-500-M with different concentration of $\mathrm{Fe}^{3+}$ is shown in Fig. 8c. The spectra indicate that the PL intensity of GQDs- $500-\mathrm{M}$ is very sensitive to the concentration of $\mathrm{Fe}^{3+}$. The PL intensity decreases with increasing $\mathrm{Fe}^{3+}$ concentration. Hence, quenching efficiency of $\mathrm{Fe}^{3+}$ was determined by $\left(\mathrm{F}_{0}-\mathrm{F}\right) /$ 

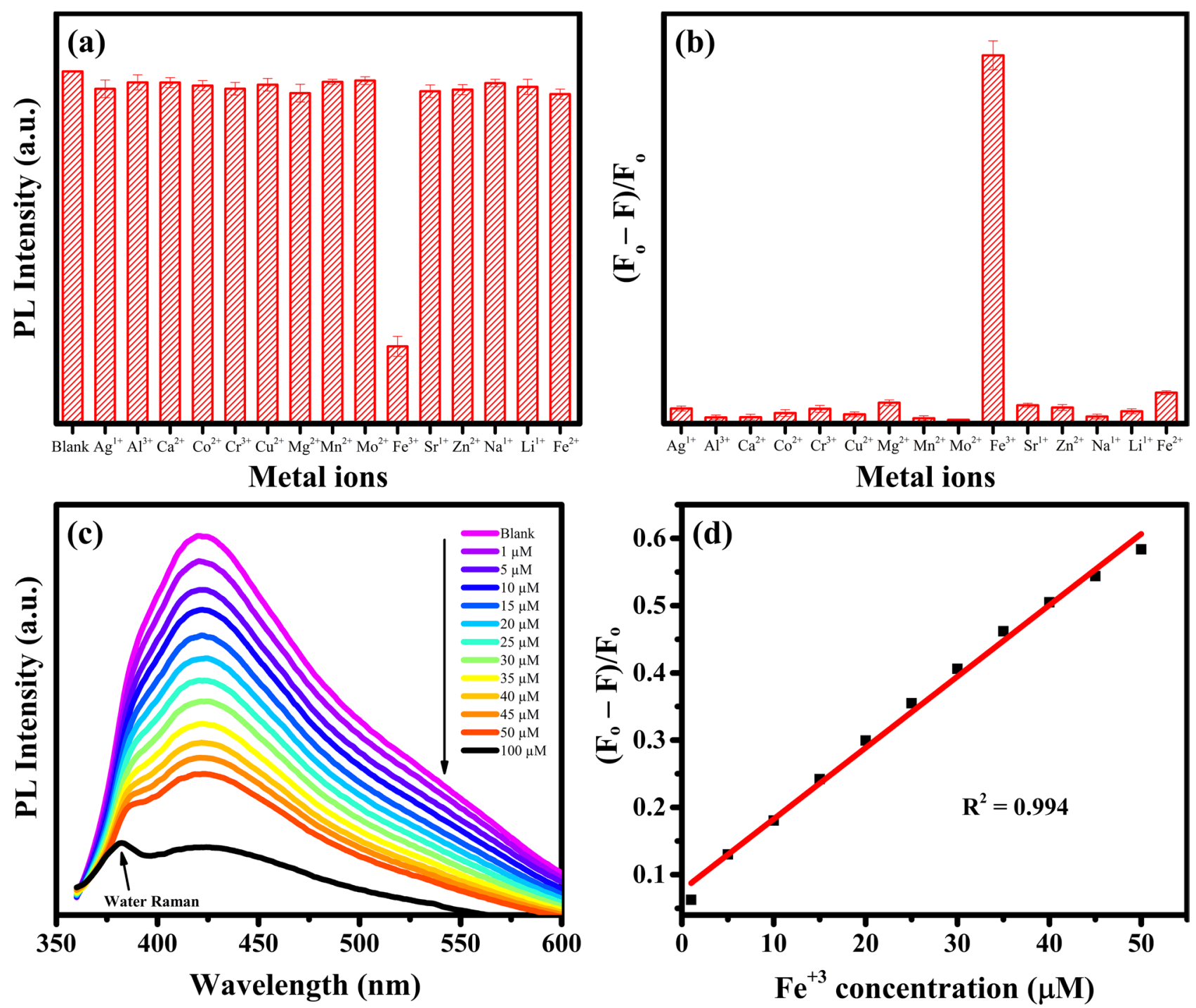

Figure 8. (a) Comparison of the PL intensities of $50 \mu \mathrm{g} \mathrm{mL} \mathrm{m}^{-1} \mathrm{GQDs}-500-\mathrm{M}$ solution in the presence of different metal ions $(100 \mu \mathrm{M})$ at an excitation wavelength of $340 \mathrm{~nm}$. (b) The comparison of the affinity of different metal ions towards GQDs-500-M ( $\mathrm{F}_{0}$ and $\mathrm{F}$ are the PL intensities of GQDs-500-M without and with $100 \mu \mathrm{M}$ of different metal ions). (c) The PL spectra of GQDs-500-M at different concentrations of $\mathrm{Fe}^{3+}$ and (d) corresponding linear plot. Three independent experiments were carried out to obtain the mean values. Figures were drawn using OriginPro 2018b (https://www.originlab.com).

$\mathrm{F}_{0}$, where $\mathrm{F}_{0}$ and $\mathrm{F}$ are the PL intensities of blank and with different concentrations of metal ions, respectively. A quick PL quenching with $\mathrm{Fe}^{3+}$ was observed, therefore the quenching test was performed at low concentration range from 1 to $50 \mu \mathrm{M}$ of $\mathrm{Fe}^{3+}$.

Meanwhile, a good linear plot is observed between the quenching efficiency and $\mathrm{Fe}^{3+}$ ions concentration $(1-50 \mu \mathrm{M})$. As shown in Fig. 8d,a linear regression value $\left(\mathrm{R}^{2}\right)$ of 0.994 was obtained which shows a good fitting and accurate detection of $\mathrm{Fe}^{3+}$ ion concentration. These results indicate that the 'as prepared' GQDs-500-M can be used as a selective and sensitive sensor for the detection of $\mathrm{Fe}^{3+}$ ions. The limit of detection (LOD) and limit of quantification (LOQ) were calculated using the following equations:

$$
\begin{aligned}
& \mathrm{LOD}=3.3 \frac{\sigma}{\mathrm{S}} \\
& \mathrm{LOQ}=10 \frac{\sigma}{\mathrm{S}}
\end{aligned}
$$

where $\sigma$ is the standard deviation of intercept and $S$ is the slop of linear regression plot.

The LOD and LOQ were calculated to be $2.5 \pm 0.3 \mu \mathrm{M}$ and $7.6 \pm 0.9 \mu \mathrm{M}$, respectively. The detection limit of $\mathrm{Fe}^{3+}$ found in this study was much lower than the World Health Organisation (WHO) drinking water guideline limit of $\mathrm{Fe}^{3+}$ concentration $(5.36 \mu \mathrm{M})^{96}$, suggesting that the as prepared GQDs-500-M are promising in detecting 


\begin{tabular}{|l|l|c|l|l|}
\hline Material & Synthesis method & Linear range $(\mu \mathrm{M})$ & LOD $(\boldsymbol{\mu M})$ & Refs. \\
\hline GQDs & Electrochemical exfoliation & $0-80$ & 7.22 & 4 \\
\hline CQDs & Thermal pyrolysis & $0-300$ & 13.68 & 97 \\
\hline CQDs & Hydrothermal & $0-20$ & 3.7 & 98 \\
\hline Carbon dots & Hydrothermal & $0-500$ & 7.4 & 99 \\
\hline N doped carbon dots & Hydrothermal & $0-50$ & 10.98 & 10 \\
\hline Carbon dots & Sonication & $12.5-100$ & 9.97 & 100 \\
\hline Carbon dots & Hydrothermal & $0-500$ & 28 & 101 \\
\hline Carbon dots & Thermal analysis & $25-300$ & 19 & 10 \\
\hline GQDs & Microwave treatment & $0-50$ & 2.5 & Present work \\
\hline
\end{tabular}

Table 3. Comparison of the previously reported work on carbon-based QDs with the present work for sensing of $\mathrm{Fe}^{3+}$ ions.

trace amounts of $\mathrm{Fe}^{3+}$. A comparison of the $\mathrm{Fe}^{3+}$ ion detection by different carbon-based materials reported by various researchers is given in Table 3. The detection limit of $2.5 \pm 0.3 \mu \mathrm{M} \mathrm{Fe}^{3+}$ observed in this work is considerably lower than the values previously reported for detecting $\mathrm{Fe}^{3+}$. Thus, we believe the 'as prepared' GQDs-500-M are very promising for $\mathrm{Fe}^{3+}$ sensing in practical applications.

\section{Conclusions}

GQDs have been successfully synthesised through microwave treatment of biomass waste derived short range ordered carbon. The sizes of as-prepared GQDs were in the range of 5 to $20 \mathrm{~nm}$ and exhibited excitation independent PL emission. This phenomenon is attributed to the single-emission fluorescence centre of these GQDs. These GQD were further modified by a simple hydrothermal treatment to reduce the particle size and enhance the optical properties. The average size of modified GQDs-500-M was $1.6 \pm 0.55 \mathrm{~nm}$, with a very narrow size distribution. The decreased size resulted in the increase of the bandgap and PL emission in the high energy region. Moreover, a high quantum yield of 0.23 was noted for GQDs-500-M. Owing to their fascinating optical properties, GQDs-500-M were applied as PL sensor to detect $\mathrm{Fe}^{3+}$ ions. These GQDs were used a fluorescence probe to selectively detect $\mathrm{Fe}^{3+}$ ions with high sensitivity down to $2.5 \pm 0.3 \mu \mathrm{M}$. Taken together, highly luminescent, cost-effective, highly soluble and sensitive sensing abilities (even at low concentrations) makes as prepared GQDs an ideal candidate for potential applications in sensing other metal ions and biomolecules.

\section{Methods}

Materials. The biomass waste, namely spent tea (PG tips), was collected from a local shop. Nitric acid $\left(\mathrm{HNO}_{3},>67 \%\right)$ and sodium hydroxide $(\mathrm{NaOH})$ were obtained from Fisher Scientific and used without modification. The salts $\mathrm{AgNO}_{3}, \mathrm{AlCl}_{3}, \mathrm{CaCl}_{2}, \mathrm{CoCl}_{2}, \mathrm{CrCl}_{3}, \mathrm{CuCl}_{2}, \mathrm{MgCl}_{2}, \mathrm{MnCl}_{2}, \mathrm{MoCl}_{2}, \mathrm{FeCl}_{3}, \mathrm{SrCl}_{2}, \mathrm{ZnCl}, \mathrm{NaCl}$, $\mathrm{LiCl}$ and $\mathrm{FeCl}_{2}$ were supplied by Sigma Aldrich. The dialysis bags of $1 \mathrm{kD}$ MWCO (Spectrum Labs) were used for purification of GQDs. Mica discs (product code: AGF7013) and TEM grids (product code: AGS147-4H) were obtained from Agar Scientific. Deionised water was used for the preparation of all solutions.

Characterisation. The structure and morphology of the samples were characterised by a wide range of analytical techniques. The size and microstructure of samples were studied by transmission electron microscope (FEI Titan Themis 300: X-FEG $300 \mathrm{kV}$ S/TEM). A software 'ImageJ (1.46r)' was used to measure the particle size from TEM images. X-ray photoelectron spectroscopy (XPS) was used to analyse the surface elemental composition on a Thermo Scientific NEXSA spectrometer. Samples were analysed using a micro focused monochromatic $\mathrm{Al}$ X-ray source $(72 \mathrm{~W})$ over an area of approximately $400 \mu \mathrm{m}$. Data were recorded at pass energies of $200 \mathrm{eV}$ for survey scans and $50 \mathrm{eV}$ for high resolution scan with $1 \mathrm{eV}$ and $0.1 \mathrm{eV}$ step sizes, respectively. Raman spectra were obtained through a Renishaw plus Raman spectrometer using $532 \mathrm{~nm}$ laser excitation. Fourier transform infrared (FTIR) spectra were recorded on a Bio-Rad FTIR spectrometer (Cary 630 FTIR, Agilent Technologies) using Diamond ATR.KBr tip. The $\mathrm{pH}$ of the samples was monitored with the help of $\mathrm{pH}$ meter (Mettler Toledo, FiveEasy $\mathrm{pH}$ meter). The absorption and fluorescence spectra were recorded on Shimadzu UV-1800 spectrophotometer and Shimadzu RF-6000 spectrofluorophotometer, respectively.

GQDs synthesis. GQDs were synthesised by microwave assisted thermochemical cutting of short range ordered carbon derived from pyrolysis of spent tea. Spent tea was washed, oven dried at $80^{\circ} \mathrm{C}$ for $12 \mathrm{~h}$ and then ground to get the fine powder $(<90 \mu \mathrm{m})$. The obtained powder was then pyrolysed at $500^{\circ} \mathrm{C}$ in inert atmosphere with a heating rate of $10^{\circ} \mathrm{C} \mathrm{min}^{-1}$ in VCTF4 furnace (Vecstar Ltd.). The pyrolysis reaction was performed for $3 \mathrm{~h}$ and carbon rich biochar was obtained. The biochar precursor was washed with DI water and then boiled in $0.1 \mathrm{M} \mathrm{HCl}$ to remove the impurities and reduce the ash content. The sample was again washed with DI water followed by drying in oven at $60{ }^{\circ} \mathrm{C}$ to get the purified product. An XPS analysis was performed to confirm purity of the precursor. The results indicated that the raw precursor was mainly composed of $\mathrm{C}, \mathrm{O}$ and $\mathrm{N}$, with traces of Ca (Fig. S2, Supplementary Information).

Next, about $20 \mathrm{mg}$ of the as prepared carbon rich sample was added into the reactor containing $10 \mathrm{ml}$ of DI water. About $2 \mathrm{ml}$ of as received $\mathrm{HNO}_{3}$ was added to the reactor to create acidic conditions for oxidative cutting 
of carbon domains. Then the reactor was treated at a microwave power of 100-900 W for 15-180 min under reflux. Upon the completion of preadjusted duration, the obtained brown dispersion was diluted with $100 \mathrm{ml}$ of DI water and filtered through $0.1 \mathrm{um}$ polyvinylidene difluoride (PVDF) filtration membranes to separate the larger unreacted particles. The obtained pale-yellow filtrate contained the GQDs and hence showed bright luminescence. The synthesised GQDs required purification due to the acidic nature of the solution. The GQDs dispersion was neutralised with $\mathrm{NaOH}$ solution until $\mathrm{pH} 7$ and then dialysed for 1 day to get the pure GQDs solution. Dry GQDs were obtained by freeze drying and a yield of up to $\sim 84.5 \mathrm{wt}$. $\%$ was recorded. The synthesised GQDs were denoted as GQDs-X, where $\mathrm{X}$ indicates the microwave power. For example, GQDs-500 denotes the product prepared at $500 \mathrm{~W}$ power.

Modification of GQDs. About $50 \mathrm{ml}$ of GQDs solution obtained before purification was added to a Teflon lined autoclave for hydrothermal treatment. After hydrothermal treatment at $200{ }^{\circ} \mathrm{C}$ for $8 \mathrm{~h}$, the dispersion was filtered through $0.1 \mu \mathrm{m}$ PVDF filtration membrane followed by purification via dialysis for 1 day. The excess water was removed using rotary evaporator. The obtained concentrated solution exhibit bright luminescence and were named as GQDs-X-M, where $\mathrm{X}$ indicates the microwave power and $\mathrm{M}$ stands for modification.

Sensing of $\mathrm{Fe}^{+3}$. The sensing of $\mathrm{Fe}^{3+}$ was performed at $340 \mathrm{~nm}$ of excitation using a normal quartz cuvette. The fluorescence spectra were measured using the mixture of GQDs-500-M and metal ions. In order to evaluate the selectivity of GQDs towards $\mathrm{Fe}^{3+}$ sensing, following fifteen different metal ions were selected: $\mathrm{Ag}^{1+}, \mathrm{Al}^{3+}$, $\mathrm{Ca}^{2+}, \mathrm{Co}^{2+}, \mathrm{Cr}^{3+}, \mathrm{Cu}^{2+}, \mathrm{Mg}^{2+}, \mathrm{Mn}^{2+}, \mathrm{Mo}^{2+}, \mathrm{Fe}^{3+}, \mathrm{Sr}^{2+}, \mathrm{Zn}^{2+}, \mathrm{Na}^{1+}, \mathrm{Li}^{1+}$ and $\mathrm{Fe}^{2+}$. The concentration of GQDs-500-M was kept constant at $50 \mu \mathrm{g} \mathrm{mL} \mathrm{m}^{-1}$ and $50 \mu \mathrm{M}$ of each metal ion was added. After mixing for $2 \mathrm{~min}$, the fluorescence spectra were measured. Quantitative measurement of $\mathrm{Fe}^{3+}$ was carried out by preparing a series of $\mathrm{FeCl}_{3}$ and GQDs-500-M solutions, in which the concentration of GQDs was kept constant but the concentration of $\mathrm{Fe}^{3+}$ varied from 0 to $50 \mu \mathrm{M}$. The fluorescence intensities of these solutions were recorded using fluorescence spectrophotometer.

\section{Data availability}

The data that supports the findings of this study are available from the corresponding author upon reasonable request.

Received: 9 August 2020; Accepted: 15 October 2020

Published online: 04 December 2020

\section{References}

1. Abbas, A., Mariana, L. T. \& Phan, A. N. Biomass-waste derived graphene quantum dots and their applications. Carbon 140, 77-99 (2018).

2. Wang, X., Sun, G., Li, N. \& Chen, P. Quantum dots derived from two-dimensional materials and their applications for catalysis and energy. Chem. Soc. Rev. 45, 2239-2262 (2016).

3. Zheng, X. T., Ananthanarayanan, A., Luo, K. Q. \& Chen, P. Glowing graphene quantum dots and carbon dots: properties, syntheses, and biological applications. Small 11, 1620-1636 (2015).

4. Ananthanarayanan, A. et al. Facile synthesis of graphene quantum dots from $3 \mathrm{D}$ graphene and their application for Fe $\mathrm{F}^{3+}$ sensing. Adv. Funct. Mater. 24, 3021-3026 (2014).

5. Zhang, Z., Zhang, J., Chen, N. \& Qu, L. Graphene quantum dots: an emerging material for energy-related applications and beyond. Energy Environ. Sci. 5, 8869-8890 (2012).

6. Ponomarenko, L. A. et al. Chaotic dirac billiard in graphene quantum dots. Science 320, 356-358 (2008).

7. Li, L.-S. \& Yan, X. Colloidal graphene quantum dots. J. Phys. Chem. Lett. 1, 2572-2576 (2010).

8. Yuan, F. et al. Engineering triangular carbon quantum dots with unprecedented narrow bandwidth emission for multicolored LEDs. Nat. Commun. 9, 2249 (2018).

9. Ye, R. et al. Bandgap engineering of coal-derived graphene quantum dots. ACS Appl. Mater. Interfaces 7, 7041-7048 (2015).

10. Khan, W. U. et al. High quantum yield green-emitting carbon dots for Fe (III) detection, biocompatible fluorescent ink and cellular imaging. Sci. Rep. 7, 1-9 (2017).

11. Xu, S., Li, D. \& Wu, P. One-pot, facile, and versatile synthesis of monolayer mos $2 /$ ws 2 quantum dots as bioimaging probes and efficient electrocatalysts for hydrogen evolution reaction. Adv. Funct. Mater. 25, 1127-1136 (2015).

12. Mitri, F., De Iacovo, A., De Luca, M., Pecora, A. \& Colace, L. Lead sulphide colloidal quantum dots for room temperature $\mathrm{NO}_{2}$ gas sensors. Sci. Rep. 10, 12556 (2020).

13. Atchudan, R., Edison, T. N. J. I., Perumal, S., Muthuchamy, N. \& Lee, Y. R. Eco-friendly synthesis of tunable fluorescent carbon nanodots from Malus floribunda for sensors and multicolor bioimaging. J. Photochem. Photobiol. A 390, 112336 (2020).

14. Park, J. et al. Graphene quantum dots: structural integrity and oxygen functional groups for high sulfur/sulfide utilization in lithium sulfur batteries. NPG Asia Mater. 8, e272-e272 (2016).

15. Mondal, S., Rana, U. \& Malik, S. Graphene quantum dot-doped polyaniline nanofiber as high performance supercapacitor electrode materials. Chem. Commun. 51, 12365-12368 (2015).

16. Lin, L. \& Zhang, S. Creating high yield water soluble luminescent graphene quantum dots via exfoliating and disintegrating carbon nanotubes and graphite flakes. Chem. Commun. 48, 10177-10179 (2012).

17. Shinde, D. B. \& Pillai, V. K. Electrochemical preparation of luminescent graphene quantum dots from multiwalled carbon nanotubes. Chem. Eur. J. 18, 12522-12528 (2012).

18. Pan, D., Zhang, J., Li, Z. \& Wu, M. Hydrothermal route for cutting graphene sheets into blue-luminescent graphene quantum dots. Adv. Mater. 22, 734-738 (2010).

19. Peng, J. et al. Graphene quantum dots derived from carbon fibers. Nano Lett. 12, 844-849 (2012).

20. Ye, R. et al. Coal as an abundants source of graphene quantum dots. Nat. Commun. 4, 2943 (2013).

21. Sangam, S. et al. Sustainable synthesis of single crystalline sulphur-doped graphene quantum dots for bioimaging and beyond. Green Chem. 20, 4245-4259 (2018).

22. Liu, Q. et al. Graphene quantum dots for energy storage and conversion: from fabrication to applications. Materials Chemistry Frontiers (2020). 
23. Wang, G. et al. Facile and highly effective synthesis of controllable lattice sulfur-doped graphene quantum dots via hydrothermal treatment of durian. ACS Appl. Mater. Interfaces (2018).

24. Li, Y. et al. An electrochemical avenue to green-luminescent graphene quantum dots as potential electron-acceptors for photovoltaics. Adv. Mater. 23, 776-780 (2011).

25. Kalita, H., Palaparthy, V. S., Baghini, M. S. \& Aslam, M. Electrochemical synthesis of graphene quantum dots from graphene oxide at room temperature and its soil moisture sensing properties. Carbon (2020).

26. Kumar, R., Kumar, V. B. \& Gedanken, A. The sonochemical synthesis of carbon dots: synthetic route, effect of parameters, and catalytic, energy, biomedical and tissue engineering applications. Ultrason. Sonochem., 105009 (2020).

27. Jiang, Z. et al. Nitrogen-doped graphene quantum dots decorated $\mathrm{ZnxCd}_{1-\mathrm{x}} \mathrm{S}$ semiconductor with tunable photoelectric properties. J. Alloys Compd. 812, 152096 (2020).

28. Shin, Y. et al. Acid-free and oxone oxidant-assisted solvothermal synthesis of graphene quantum dots using various natural carbon materials as resources. Nanoscale 7, 5633-5637 (2015).

29. Kang, S. et al. Pulsed laser ablation based synthetic route for nitrogen-doped graphene quantum dots using graphite flakes. Appl. Surf. Sci. 506, 144998 (2020).

30. Li, L. L. et al. A facile microwave avenue to electrochemiluminescent two-color graphene quantum dots. Adv. Funct. Mater. 22, 2971-2979 (2012)

31. Dager, A., Baliyan, A., Kurosu, S., Maekawa, T. \& Tachibana, M. Ultrafast synthesis of carbon quantum dots from fenugreek seeds using microwave plasma enhanced decomposition: application of C-QDs to grow fluorescent protein crystals. Sci. Rep. 10, $12333(2020)$.

32. Luo, Z. et al. Microwave-assisted preparation of white fluorescent graphene quantum dots as a novel phosphor for enhanced white-light-emitting diodes. Adv. Funct. Mater. 26, 2739-2744 (2016).

33. Shin, Y. et al. Mass production of graphene quantum dots by one-pot synthesis directly from graphite in high yield. Small 10, 866-870 (2014).

34. Nair, R. V. et al. Rapid, acid-free synthesis of high-quality graphene quantum dots for aggregation induced sensing of metal ions and bioimaging. ACS Omega 2, 8051-8061 (2017).

35. Rurack, K., Kollmannsberger, M., Resch-Genger, U. \& Daub, J. A selective and sensitive fluoroionophore for HgII, AgI, and CuII with virtually decoupled fluorophore and receptor units. J. Am. Chem. Soc. 122, 968-969 (2000).

36. Shtepliuk, I. et al. On the interaction of toxic Heavy Metals $(\mathrm{Cd}, \mathrm{Hg}, \mathrm{Pb})$ with graphene quantum dots and infinite graphene. Sci. Rep. 7, 3934 (2017).

37. Zhang, M. et al. Facile synthesis of water-soluble, highly fluorescent graphene quantum dots as a robust biological label for stem cells. J. Mater. Chem. 22, 7461-7467 (2012).

38. Dong, Y. et al. Graphene quantum dot as a green and facile sensor for free chlorine in drinking water. Anal. Chem. 84, 8378-8382 (2012).

39. Suryawanshi, A. et al. Large scale synthesis of graphene quantum dots (GQDs) from waste biomass and their use as an efficient and selective photoluminescence on-off-on probe for Ag+ ions. Nanoscale 6, 11664-11670 (2014).

40. Bai, J. M., Zhang, L., Liang, R. P. \& Qiu, J. D. Graphene quantum dots combined with europium ions as photoluminescent probes for phosphate sensing. Chem. Eur. J. 19, 3822-3826 (2013).

41. Wang, C. et al. Graphene quantum dots nanosensor derived from 3D nanomesh graphene frameworks and its application for fluorescent sensing of $\mathrm{Cu}^{2+}$ in rat brain. Sens. Actuators B Chem. 258, 672-681 (2018).

42. Salehnia, F., Faridbod, F., Dezfuli, A. S., Ganjali, M. R. \& Norouzi, P. Cerium (III) ion sensing based on graphene quantum dots fluorescent turn-off. J. Fluoresc. 27, 331-338 (2017).

43. Wang, D., Wang, L., Dong, X., Shi, Z. \& Jin, J. Chemically tailoring graphene oxides into fluorescent nanosheets for $\mathrm{Fe}^{3+}$ ion detection. Carbon 50, 2147-2154 (2012).

44. Mounsey, R. B. \& Teismann, P. Chelators in the treatment of iron accumulation in Parkinson's disease. Int. J. Cell Biol. 2012 (2012).

45. Zhu, X. et al. Understanding the selective detection of $\mathrm{Fe}^{3+}$ based on graphene quantum dots as fluorescent probes: the $\mathrm{K}$ sp of a metal hydroxide-assisted mechanism. Anal. Chem. 89, 12054-12058 (2017).

46. Gao, X. et al. Exonuclease I-assisted general strategy to convert aptamer-based electrochemical biosensors from "signal-off" to "signal-on". Anal. Chem. (2020).

47. Zhang, J. F., Zhou, Y., Yoon, J. \& Kim, J. S. Recent progress in fluorescent and colorimetric chemosensors for detection of precious metal ions (silver, gold and platinum ions). Chem. Soc. Rev. 40, 3416-3429 (2011).

48. Luo, Z., Lu, Y., Somers, L. A. \& Johnson, A. C. High yield preparation of macroscopic graphene oxide membranes. J. Am. Chem. Soc. 131, 898-899 (2009).

49. Aidas, K., Kongsted, J., Osted, A., Mikkelsen, K. V. \& Christiansen, O. Coupled cluster calculation of the $\mathrm{n} \rightarrow \pi^{*}$ electronic transition of acetone in aqueous solution. J. Phys. Chem. A 109, 8001-8010 (2005).

50. Sun, Y. et al. Magnetism of graphene quantum dots. NPJ Quant. Mater. 2, 1-7 (2017).

51. Tauc, J., Grigorovici, R. \& Vancu, A. Optical properties and electronic structure of amorphous germanium. Phys. Status Solidi $B$ 15, 627-637 (1966).

52. He, S., Turnbull, M. J., Nie, Y., Sun, X. \& Ding, Z. Band structures of blue luminescent nitrogen-doped graphene quantum dots by synchrotron-based XPS. Surf. Sci. 676, 51-55 (2018).

53. Das, R., Sugimoto, H., Fujii, M. \& Giri, P. K. Quantitative understanding of charge transfer mediated $\mathrm{Fe}^{3+}$ sensing and fast photoresponse by $\mathrm{n}$-doped graphene quantum dots decorated on plasmonic Au nanoparticles. ACS Appl. Mater. Interfaces (2020).

54. Yan, Y. et al. Systematic bandgap engineering of graphene quantum dots and applications for photocatalytic water splitting and $\mathrm{CO}_{2}$ reduction. ACS Nano 12, 3523-3532 (2018).

55. Wu, X. et al. A versatile platform for the highly efficient preparation of graphene quantum dots: photoluminescence emission and hydrophilicity-hydrophobicity regulation and organelle imaging. Nanoscale 10, 1532-1539 (2018).

56. Tetsuka, H. et al. Optically tunable amino-functionalized graphene quantum dots. Adv. Mater. 24, 5333-5338 (2012)

57. Zhu, S. et al. Strongly green-photoluminescent graphene quantum dots for bioimaging applications. Chem. Commun. 47, 6858$6860(2011)$.

58. Sun, J. et al. Ultra-high quantum yield of graphene quantum dots: aromatic-nitrogen doping and photoluminescence mechanism. Part. Part. Syst. Char. 32, 434-440 (2015).

59. Zhu, S. et al. Surface chemistry routes to modulate the photoluminescence of graphene quantum dots: from fluorescence mechanism to up-conversion bioimaging applications. Adv. Funct. Mater. 22, 4732-4740 (2012).

60. Ding, H., Yu, S.-B., Wei, J.-S. \& Xiong, H.-M. Full-color light-emitting carbon dots with a surface-state-controlled luminescence mechanism. ACS Nano 10, 484-491 (2015).

61. Wunsch, B., Stauber, T. \& Guinea, F. Electron-electron interactions and charging effects in graphene quantum dots. Phys. Rev. B 77, 035316 (2008).

62. Yang, P. et al. Facile synthesis and photoluminescence mechanism of graphene quantum dots. J. Appl. Phys. 116, 244306 (2014).

63. Zhang, F. et al. Effect of lateral size of graphene quantum dots on their properties and application. ACS Appl. Mater. Interfaces 8, 2104-2110 (2016) 
64. Bian, S. et al. Facile synthesis of sulfur-doped graphene quantum dots as fluorescent sensing probes for Ag+ ions detection. Sens. Actuators B Chem. 242, 231-237 (2017).

65. Wang, L. et al. Gram-scale synthesis of single-crystalline graphene quantum dots with superior optical properties. Nat. Commun. 5, 1-9 (2014).

66. Maiti, S., Kundu, S., Roy, C. N., Das, T. K. \& Saha, A. Synthesis of excitation independent highly luminescent graphene quantum dots through perchloric acid oxidation. Langmuir 33, 14634-14642 (2017).

67. Ye, R. et al. Coal as an abundant source of graphene quantum dots. Nat. Commun. 4, 2943 (2013).

68. Joffrion, J. B., Clower, W. \& Wilson, C. G. Tunable excitation-independent emissions from graphene quantum dots through microplasma-assisted electrochemical synthesis. Nano Struct. Nano Objects 19, 100341 (2019).

69. Roy, P. et al. Plant leaf-derived graphene quantum dots and applications for white LEDs. New J. Chem. 38, 4946-4951 (2014).

70. Wang, R. et al. Direct transformation of lignin into fluorescence-switchable graphene quantum dots and their application in ultrasensitive profiling of a physiological oxidant. Green Chem. 21, 3343-3352 (2019).

71. Wang, Z. et al. Large-scale and controllable synthesis of graphene quantum dots from rice husk biomass: a comprehensive utilization strategy. ACS Appl. Mater. Interfaces 8, 1434-1439 (2016).

72. Wang, L. et al. Facile synthesis of fluorescent graphene quantum dots from coffee grounds for bioimaging and sensing. Chem. Eng. J. 300, 75-82 (2016).

73. Ding, Z., Li, F., Wen, J., Wang, X. \& Sun, R. Gram-scale synthesis of single-crystalline graphene quantum dots derived from lignin biomass. Green Chem. 20, 1383-1390 (2018).

74. Zhao, S. et al. Green synthesis of bifunctional fluorescent carbon dots from garlic for cellular imaging and free radical scavenging. ACS Appl. Mater. Interfaces 7, 17054-17060 (2015).

75. Liu, W. et al. Highly crystalline carbon dots from fresh tomato: UV emission and quantum confinement. Nanotechnology 28, 485705 (2017).

76. Adinarayana, L. et al. Single step synthesis of carbon quantum dots from coconut shell: evaluation for antioxidant efficacy and hemotoxicity. J. Mater. Sci. Appl 3, 83-93 (2017).

77. Liang, Z., Kang, M., Payne, G. F., Wang, X. \& Sun, R. Probing energy and electron transfer mechanisms in fluorescence quenching of biomass carbon quantum dots. ACS Appl. Mater. Interfaces 8, 17478-17488 (2016).

78. Liu, J. et al. Deep red emissive carbonized polymer dots with unprecedented narrow full width at half maximum. Adv. Mater. 32, 1906641 (2020).

79. Dai, Y. et al. Versatile graphene quantum dots with tunable nitrogen doping. Part. Part. Syst. Char. 31, 597-604 (2014).

80. Hasan, M. T. et al. Photo-and electroluminescence from nitrogen-doped and nitrogen-sulfur codoped graphene quantum dots. Adv. Funct. Mater. 28, 1804337 (2018).

81. Kim, S. et al. Size-dependence of Raman scattering from graphene quantum dots: interplay between shape and thickness. Appl. Phys. Lett. 102, 053108 (2013).

82. Ferrari, A. C. \& Basko, D. M. Raman spectroscopy as a versatile tool for studying the properties of graphene. Nat. Nanotechnol. 8, 235-246 (2013).

83. Tuinstra, F. \& Koenig, J. L. Raman spectrum of graphite. J. Chem. Phys. 53, 1126-1130 (1970).

84. Ferrari, A. C. \& Robertson, J. Interpretation of Raman spectra of disordered and amorphous carbon. Phys. Rev. B 61, 14095 (2000).

85. He, X., Zhang, S., Pan, H., Chen, J. \& Xu, J. Horizontally aggregation of monolayer reduced graphene oxide under deep uv irradiation in solution. Nanoscale Res. Lett. 14, 117 (2019).

86. Hu, C. et al. One-step preparation of nitrogen-doped graphene quantum dots from oxidized debris of graphene oxide. J. Mater. Chem. B 1, 39-42 (2013).

87. Dai, L. et al. Calcium-rich biochar from the pyrolysis of crab shell for phosphorus removal. J. Environ. Manag. 198, 70-74 (2017).

88. Pels, J. R., Kapteijn, F., Moulijn, J. A., Zhu, Q. \& Thomas, K. M. Evolution of nitrogen functionalities in carbonaceous materials during pyrolysis. Carbon 33, 1641-1653 (1995).

89. Sevilla, M. \& Fuertes, A. B. Chemical and structural properties of carbonaceous products obtained by hydrothermal carbonization of saccharides. Chem. Eur. J. 15, 4195-4203 (2009).

90. Deka, M. J., Dutta, A. \& Chowdhury, D. Tuning the wettability and photoluminescence of graphene quantum dots via covalent modification. New J. Chem. 42, 355-362 (2018).

91. Hsu, P.-C. \& Chang, H.-T. Synthesis of high-quality carbon nanodots from hydrophilic compounds: role of functional groups. Chem. Commun. 48, 3984-3986 (2012).

92. Lesani, P. et al. Two-photon dual-emissive carbon dot-based probe: deep-tissue imaging and ultrasensitive sensing of intracellular ferric ions. ACS Appl. Mater. Interfaces 12, 18395-18406 (2020).

93. Dutta Chowdhury, A. \& Doong, R.-A. Highly sensitive and selective detection of nanomolar ferric ions using dopamine functionalized graphene quantum dots. ACS Appl. Mater. Interfaces 8, 21002-21010 (2016).

94. Sun, X. \& Lei, Y. Fluorescent carbon dots and their sensing applications. TrAC Trends Anal. Chem. 89, 163-180 (2017).

95. Lesani, P. et al. Two-photon dual-emissive carbon dot-based probe: deep tissue imaging and ultrasensitive sensing of intracellular ferric ions. ACS Appl. Mater. Interfaces (2020).

96. World Health Organization. Guidelines for Drinking-Water Quality (World Health Organization, Geneva, 1993).

97. Xie, Z., Sun, X., Jiao, J. \& Xin, X. Ionic liquid-functionalized carbon quantum dots as fluorescent probes for sensitive and selective detection of iron ion and ascorbic acid. Colloids Surf. A 529, 38-44 (2017).

98. Wu, F., Su, H., Wang, K., Wong, W.-K. \& Zhu, X. Facile synthesis of N-rich carbon quantum dots from porphyrins as efficient probes for bioimaging and biosensing in living cells. Int. J. Nanomed. 12, 7375 (2017).

99. Wang, Z., Long, P., Feng, Y., Qin, C. \& Feng, W. Surface passivation of carbon dots with ethylene glycol and their high-sensitivity to $\mathrm{Fe}^{3+}$. RSC Adv. 7, 2810-2816 (2017).

100. Aslandaş, A. M. et al. Liquid nitrogen-assisted synthesis of fluorescent carbon dots from Blueberry and their performance in $\mathrm{Fe}^{3+}$ detection. Appl. Surf. Sci. 356, 747-752 (2015)

101. Li, Q. et al. Induction of long-lived room temperature phosphorescence of carbon dots by water in hydrogen-bonded matrices. Nat. Commun. 9, 1-8 (2018).

\section{Acknowledgements}

The authors gratefully acknowledge the financial support from the Faculty of Science, Agriculture \& Engineering (SAgE), School of Engineering, and International Fund Partnership Newcastle University for their financial support (OSR/0530/SD16/ABBA).

\section{Author contributions}

A.A. designed the project, carried out all the experiments and wrote the manuscript. T.A.T. contributed to the characterisations and revised the manuscript. S.J.B., T.M.L. and A.N.P supervised the project and reviewed the manuscript. All authors approved the final manuscript. 


\section{Competing interests}

The authors declare no competing interests.

\section{Additional information}

Supplementary information is available for this paper at https://doi.org/10.1038/s41598-020-78070-2.

Correspondence and requests for materials should be addressed to T.M.L. or A.N.P.

Reprints and permissions information is available at www.nature.com/reprints.

Publisher's note Springer Nature remains neutral with regard to jurisdictional claims in published maps and institutional affiliations.

(c) (i) Open Access This article is licensed under a Creative Commons Attribution 4.0 International cc) License, which permits use, sharing, adaptation, distribution and reproduction in any medium or format, as long as you give appropriate credit to the original author(s) and the source, provide a link to the Creative Commons licence, and indicate if changes were made. The images or other third party material in this article are included in the article's Creative Commons licence, unless indicated otherwise in a credit line to the material. If material is not included in the article's Creative Commons licence and your intended use is not permitted by statutory regulation or exceeds the permitted use, you will need to obtain permission directly from the copyright holder. To view a copy of this licence, visit http://creativecommons.org/licenses/by/4.0/.

(C) The Author(s) 2020 